DOI 10.4171/JEMS/366

Robert Juhlin · Bernhard Lamel

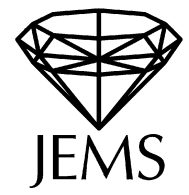

\title{
Automorphism groups of minimal real-analytic CR manifolds
}

Received November 23, 2010 and in revised form October 25, 2011

Abstract. We show that the local automorphism group of a minimal real-analytic CR manifold $M$ is a finite-dimensional Lie group if (and only if) $M$ is holomorphically nondegenerate by constructing a jet parametrization.

\section{Introduction}

In 1907, Poincaré observed that it is extremely unlikely that one can find an analytic map between two given real hypersurfaces in $\mathbb{C}^{2}$; in his paper, he formulated the "problème local": Given two hypersurfaces, is it possible to determine all such maps? In this paper, we shall answer the analogous question for automorphisms of real-analytic CR manifolds; we establish a necessary and sufficient condition (known as holomorphic nondegeneracy) for the finite-dimensionality of the automorphism groups and furthermore give an algorithm to compute them. We note that this problem is markedly different from the analogous problem in the complex plane, where it is well known that two real-analytic arcs can always be mapped into each other by an analytic map, and the space of such maps is necessarily of infinite dimension.

The first positive answer to this question was given by Élie Cartan in 1932 [6, 7] for strictly pseudoconvex real-analytic hypersurfaces in $\mathbb{C}^{2}$, by applying his method of equivalence. This type of result, which establishes a complete list of invariants which can be used to solve the local equivalence problem, is today known for Levi-nondegenerate real-analytic hypersurfaces in $\mathbb{C}^{N}$, by the work of Tanaka [17] and Chern and Moser [8], as well as for minimal real-analytic hypersurfaces in $\mathbb{C}^{2}$ by the work of Kolár [14]. An important corollary to all of their works is that the local automorphism group of such a real-analytic hypersurface is a subgroup of the automorphism group of the hyperquadric (or, in Koláŕ's case, a more general model); these groups, however, are well known, and turn out to be finite-dimensional Lie groups.

The methods used to prove the results for Levi-nondegenerate real-analytic hypersurfaces do not carry over to degenerate cases, and Koláŕ's techniques only extend to very

R. Juhlin, B. Lamel: Fakultät für Mathematik, Universität Wien, Nordbergstrasse 15, A-1090 Wien, Austria; e-mail: robert.juhlin@univie.ac.at, bernhard.lamel@univie.ac.at

Mathematics Subject Classification (2010): 32H02, 32V40 
specific cases in higher dimensions [13]. However, Poincaré's observation was very general, and leads us to expect that even though we might not be able to give a complete list of invariants, there should not be "too many" maps; maybe we can even prove that the automorphism groups of more general real-analytic submanifolds are finite-dimensional Lie groups?

Some partial positive answers to these questions have been given over the last decade. The first results valid for Levi-degenerate real-analytic hypersurfaces, due to Baouendi, Ebenfelt, and Rothschild [2] and generalized to higher codimension by Zaitsev [18], gave not only a solution to the problem in the "finitely nondegenerate" case, but also a general way of attacking the problem through so-called jet parametrizations. In the context of automorphism groups of germs of real-analytic submanifold $M \subset \mathbb{C}^{N}$ at a distinguished point $p \in M$, a jet parametrization inverts the natural mapping $\operatorname{Aut}(M, p) \rightarrow G_{p}^{k}\left(\mathbb{C}^{N}\right)$ from the local automorphism group

$$
\begin{array}{r}
\operatorname{Aut}(M, p)=\left\{H:\left(\mathbb{C}^{N}, p\right) \rightarrow\left(\mathbb{C}^{N}, p\right): \operatorname{det} H^{\prime}(p) \neq 0, H(M \cap U) \subset M\right. \\
\text { for some neighbourhood } U \text { of } p\}
\end{array}
$$

of germs of biholomorphisms of $\left(\mathbb{C}^{N}, p\right)$ mapping $M$ to itself endowed with its natural inductive limit topology, to the jet group of order $k$ at the point $p$ for some $k$. It thus provides a mapping $\Psi$ defined near $\{p\} \times \mathcal{U}$ for some $\mathcal{U} \subset G_{p}^{k}\left(\mathbb{C}^{N}\right)$ with

$$
\Psi\left(Z, j_{p}^{k} H\right)=H(Z), \quad H \in \operatorname{Aut}(M, p) .
$$

A jet parametrization which is in addition holomorphic in the first argument and realanalytic in the second shows that the image of the natural embedding of a neighbourhood of, say, the identity in $\operatorname{Aut}(M, p)$ as a neighbourhood of the identity in a subgroup of $G_{p}^{k}\left(\mathbb{C}^{N}\right)$ can be defined by real-analytic equations and thus endows $\operatorname{Aut}(M, p)$ with a Lie-group structure.

Jet parametrizations have turned out to be powerful tools. For minimal real-analytic hypersurfaces in $\mathbb{C}^{2}$, a jet parametrization was used by Ebenfelt, Zaitsev and the second author [10] to provide a solution of our question above; Mir and the second author [15] used it to prove that $\operatorname{Aut}(M, p)$ is a finite-dimensional Lie group for any real-analytic CR manifold $M$ which is minimal at $p$ and "essentially finite".

All of the results discussed so far provide sufficient conditions for $\operatorname{Aut}(M, p)$ to be finite-dimensional; in order to see what a necessary condition looks like, it is helpful to look at the Lie algebra of infinitesimal automorphisms. An infinitesimal automorphism of $(M, p)$ is a germ of a holomorphic $(1,0)$-vector field

$$
X=\sum_{j=1}^{N} a_{j}(Z) \frac{\partial}{\partial Z_{j}},
$$

vanishing at $p$, whose real part is tangent to $M$; we denote the space of infinitesimal automorphisms of $(M, p)$ by hol $(M, p)$. Clearly, if $X$ itself is tangent to $M$ near $p$, then $X \in \mathfrak{h o l}(M, p)$; we shall denote the subspace of all such $X$ by $\mathfrak{h t}(M, p) \subset \mathfrak{h o l}(M, p)$ and refer to them as tangent holomorphic vector fields. 
It is easy to see that if $\mathfrak{h t}(M, p) \neq\{0\}$, then necessarily $\operatorname{dim}_{\mathbb{C}} \mathfrak{h} \mathfrak{t}(M, p)=\infty$ (after all, $\mathfrak{h} \mathfrak{t}(M, p)$ is a module over the ring of germs of holomorphic functions at 0$)$, which in turn precludes that $\operatorname{Aut}(M, p)$ can be given the structure of a finite-dimensional Lie group. Thus, $\mathfrak{h} \mathfrak{t}(M, p)=\{0\}$ is clearly necessary for $\operatorname{Aut}(M, p)$ to be finite-dimensional. We say that a connected CR manifold $M$ is holomorphically nondegenerate if $\mathfrak{h t}(M, p)=\{0\}$ for one (or, equivalently, for all) $p \in M$. Holomorphic nondegeneracy was introduced by Stanton [16] and shown to be sufficient for $\mathfrak{h o l}(M, p)$ to be finite-dimensional by Baouendi, Ebenfelt, and Rothschild [3]. In this paper we show that actually $\operatorname{Aut}(M, p)$ is a finite-dimensional Lie group for all minimal holomorphically nondegenerate CR manifolds, thus showing that holomorphic nondegeneracy characterizes the finite-dimensionality of $\operatorname{Aut}(M, p)$ :

Theorem 1. Let $M$ be a connected real-analytic $C R$ manifold which is minimal at $p \in$ $M$. Then the group $\operatorname{Aut}(M, p)$ of real-analytic $C R$ automorphisms of $(M, p)$ is a finitedimensional Lie group if and only if $M$ is holomorphically nondegenerate. Furthermore, in this case, $\operatorname{Aut}(M, p)$ can be identified with a real algebraic subgroup of the jet group $G_{p}^{k}(M)$ for some $k \in \mathbb{N}$.

Let us recall that $M$ is minimal at $p \in M$ if there is no proper CR submanifold $N \subset M$ containing $p$ of the same CR dimension as $M$. We shall establish Theorem 1 by constructing a jet parametrization for automorphisms of a holomorphically nondegenerate minimal CR manifold. Before we state our jet parametrization theorem, let us note the following interesting corollary of Theorem 1 .

Corollary 1. Let $M$ be a connected, minimal real-analytic $C R$ manifold. If $\operatorname{Aut}(M, p)$ is a finite-dimensional Lie group for some $p \in M$, then $\operatorname{Aut}(M, q)$ is a finite-dimensional Lie group for all $q \in M$.

Indeed, as we already noted above, a real-analytic CR manifold has the property that if $\mathfrak{h t}(M, p)=\{0\}$ for some $p \in M$, then $\mathfrak{h} \mathfrak{t}(M, q)=\{0\}$ for all $q$ in the connected component of $M$ containing $p$.

Let us now state our main parametrization theorem.

Theorem 2. Let $(M, p) \subset\left(\mathbb{C}^{N}, p\right)$ be a germ of a generic real analytic submanifold of $\mathbb{C}^{N}$, which is minimal and holomorphically nondegenerate at $p$. Then there exists $k \in \mathbb{N}$, a neighbourhood $U \subset G_{p}^{k}\left(\mathbb{C}^{N}\right)$ of $j_{p}^{k} \mathrm{id}$, and a map $\Psi: \mathcal{U} \rightarrow \mathbb{C}^{N}$, defined on a neighbourhood $\mathcal{U}$ of $\{p\} \times U$ in $\mathbb{C}^{N} \times G_{p}^{k}\left(\mathbb{C}^{N}\right)$, holomorphic in the first variable and real-analytic in the second, such that

$$
H(Z)=\Psi\left(Z, j_{p}^{k} H\right) \quad \text { for all } H \in \operatorname{Aut}(M, p) \text { with } j_{p}^{k} H \in U .
$$

Furthermore, there exists a polynomial s with $s\left(j_{p}^{k} \mathrm{id}\right) \neq 0$ and for every $\alpha \in \mathbb{N}^{N}$ a polynomial $p_{\alpha}$ on $G_{p}^{k}\left(\mathbb{C}^{N}\right)$ and an integer $r_{\alpha}$ such that

$$
\Psi(Z, \Lambda)=\sum_{\alpha \in \mathbb{N}^{N}} \frac{p_{\alpha}(\Lambda)}{s(\Lambda)^{r_{\alpha}}} Z^{\alpha} .
$$


Furthermore, the integer $k=k(M, p)$ can be bounded by local biholomorphic invariants of $(M, p)$ and can be chosen in such a way that it remains uniformly bounded on compact subsets of $M$.

Let us quickly review how a parametrization as in (1) lets us identify the image of $j_{p}^{k}: \operatorname{Aut}(M, p) \rightarrow G_{p}^{k}\left(\mathbb{C}^{N}\right)$. Using a real-analytic defining function $\rho(Z, \bar{Z})$ of $M$ and a real-analytic parametrization $\mathbb{R}^{2 N-d} \ni \xi \mapsto \varphi(\xi)$ of $M$, we see that $\Lambda \in j_{p}^{k}(\operatorname{Aut}(M, p))$ if and only if

$$
0=\rho(\Psi(\varphi(\xi), \Lambda), \overline{\Psi(\varphi(\xi), \Lambda)})=\sum_{\beta} \frac{P_{\beta}(\Lambda)}{Q(\Lambda)^{d_{\beta}}} \xi^{\beta}
$$

and thus $j_{p}^{k}(\operatorname{Aut}(M, p))$ can be defined by the (real) polynomial equations $P_{\beta}(\Lambda)=0$. This argument shows that Theorem 1 follows from Theorem 2, since it shows that a neighbourhood of the identity in $\operatorname{Aut}(M, p)$ is homeomorphic to a real-algebraic subset of a neighbourhood of the identity in $G_{p}^{k}\left(\mathbb{C}^{N}\right)$. It actually yields the following, somewhat more explicit theorem, which identifies $\operatorname{Aut}(M, p)$ with a subgroup of $G_{p}^{k}\left(\mathbb{C}^{N}\right)$ :

Theorem 3. Let $M$ be the germ of a generic real-analytic submanifold of $\mathbb{C}^{N}$ at $p$. Then there exists an integer $k=k(p)$ such that the natural map $j_{p}^{k}: \operatorname{Aut}(M, p) \rightarrow G_{p}^{k}\left(\mathbb{C}^{N}\right)$ is an embedding which is a homeomorphism onto its image, which is a real-algebraic closed subgroup of $G_{p}^{k}\left(\mathbb{C}^{N}\right)$. Furthermore, the integer $k(p)$ can be chosen in such a way that it remains uniformly bounded on compact subsets of $M$.

A typical application of a finite jet parametrization only uses that $H \in \operatorname{Aut}(M, p)$ is uniquely determined by $j_{0}^{k} H$. In our setting, this unique determination property is due to Baouendi, Mir and Rothschild [5], and in more generality to the first author [12] who both actually proved it for mappings which are not necessarily automorphisms. We now state a uniqueness property which follows from our theorem.

Corollary 2. Let $M$ be a real-analytic, holomorphically nondegenerate minimal $C R$ manifold. Then for any compact subset $K \subset M$ there exists an integer $k=k(K)$ such that if $H$ and $\tilde{H}$ are real-analytic $C R$ automorphisms defined in a neighbourhood of $p \in M$ with $j_{p}^{k} H=j_{p}^{k} \tilde{H}$, then $H=\tilde{H}$.

A further implication of our Theorem 2 is a global statement. For a general real-analytic CR manifold $M$, it is well known that for $p$ outside a real-analytic subvariety $V$, the germ $(M, p)$ is biholomorphically equivalent to $\left(\tilde{M} \times \mathbb{C}^{s}, 0\right)$ where $\tilde{M}$ is a germ of a holomorphically nondegenerate submanifold of $\mathbb{C}^{N-s}$ through 0 . To be exact, the sections of the sheaf $\mathfrak{h t}(M)$ with stalks $\mathfrak{h t}(M, p)$ defined above define a foliation of $M$ by holomorphic manifolds which we refer to as the holomorphic foliation of $M$. Any biholomorphism of $M$ has to preserve leaves of this foliation. Let us denote by $V$ the singular set of this foliation. At points $p \in M \backslash V$, in terms of the local product decomposition $(M, p) \cong\left(\tilde{M} \times \mathbb{C}^{s}, 0\right)$, any $H \in \operatorname{Aut}\left(\tilde{M} \times \mathbb{C}^{s}, 0\right)$ can be written as $H=\left(H_{1}, H_{2}\right)$ where $H_{1} \in \operatorname{Aut}(\tilde{M}, 0)$ and $H_{2}$ is any map of the form $H_{2}\left(Z_{1}, Z_{2}\right)$ with $\operatorname{det} \frac{\partial H_{2}}{\partial Z_{2}}(0) \neq 0$. Of course, the set of these maps does not form a finite-dimensional Lie group, but we can 
show that they form an infinite-dimensional Lie group, where we shall define a Lie group to be a group which is a locally convex manifold (actually, our groups are modelled on spaces of germs of power series) whose multiplication and inversion are analytic (in the usual infinite-dimensional sense, i.e. analytic along finite-dimensional subsets and continuous in the vector space topology). The groups which appear here will be subgroups of the group of germs of biholomorphisms of $\mathbb{C}^{N}$, which is a Lie group in this sense.

To discuss the structure of $\operatorname{Aut}\left(\tilde{M} \times \mathbb{C}^{s}, 0\right)$, we use the product decomposition $H=\left(H_{1}, H_{2}\right)$ where $H_{1} \in \operatorname{Aut}(\tilde{M}, 0)$ and $H_{2}$ is any map of the form $H_{2}\left(Z_{1}, Z_{2}\right)$ with det $\frac{\partial H_{2}}{\partial Z_{2}}(0) \neq 0$. Equivalently, $H_{2}$ is a germ of a holomorphic map $\left(\mathbb{C}^{N-s}, 0\right) \rightarrow \operatorname{Aut}\left(\mathbb{C}^{s}, 0\right)$. The set of all such germs, which we denote by $\mathcal{H}_{s}=$ $\mathcal{H}\left(\left(\mathbb{C}^{N-s}, 0\right)\right.$, Aut $\left.\left(\mathbb{C}^{s}, 0\right)\right)$, inherits the structure of an infinite-dimensional Lie group from $\operatorname{Aut}\left(\mathbb{C}^{s}, 0\right)$. Furthermore, $\operatorname{Aut}\left(\mathbb{C}^{N-s}, 0\right)$ acts on $\mathcal{H}_{s}$ in an obvious way. The group of all germs of biholomorphisms of $\mathbb{C}^{N}$ of the form $\left(Z_{1}, Z_{2}\right) \mapsto\left(h_{1}\left(Z_{1}\right), h_{2}\left(Z_{1}, Z_{2}\right)\right)$ is then exactly the semidirect product $\operatorname{Aut}\left(\mathbb{C}^{N-s}, 0\right) \ltimes \mathcal{H}_{s}$.

At a point where the holomorphic foliation is nonsingular, we can thus explicitly describe the infinite-dimensional part of the automorphism group of a general real-analytic CR manifold at all points $p \in M \backslash V$ where $V$ is the real-analytic subvariety defined before.

Theorem 4. Let $M$ be a real-analytic, minimal $C R$ manifold. Then there exists an integer $s$ such that for the subvariety $V \subset M$ defined before, for all $p \in M \backslash V$ there exists an integer $k$ such that $\operatorname{Aut}(M, p)=F \ltimes \mathcal{H}_{s}$ for a finite-dimensional Lie-group $F=$ $F(p)$ with the property that $F(p)$ embeds into the jet group $G_{p}^{k}\left(\mathbb{C}^{N}\right)$ as a closed, realalgebraic subgroup. In particular, $\operatorname{Aut}(M, p)$ is an (infinite-dimensional) Lie group for every $p \in M \backslash V$.

We now outline the proof of our main result. The main technical innovation of this paper is a new kind of parametrization theorem (Theorem 7) for solutions of singular systems of analytic equations. It replaces the corresponding result used in [15] for the situation studied here and allows us to "invert" a certain system of equations for our map associated to a holomorphically nondegenerate submanifold. This inversion is only valid for maps which are on a stratum where a certain vanishing order is constant; we relate this vanishing order to local invariants of $(M, p)$. These invariants are introduced in $\S 3$ and their basic properties are investigated. This step, which is in essence based on mapping formulas from [12], allows us to construct jet parametrizations "along the Segre maps" (see Corollary 14); we thus obtain a parametrization of the form $H(S(x))=\Psi\left(x, j_{p}^{k} H\right)$, where $S$ is a holomorphic submersion.

In order to pass from this to a parametrization as in Theorem 2, we have to use another form of inversion. While a technique to overcome this problem is available in the literature (dubbed as the "doubling trick"), we have decided to attack this problem in a more direct way, and thus include the relevant theorem, which is another new result of this paper (Theorem 5): We introduce an inversion formula for the mapping induced by composition with the germ of a holomorphic map $A:\left(\mathbb{C}^{m}, 0\right) \rightarrow\left(\mathbb{C}^{n}, 0\right)$ which is generically of full rank $n$ on the spaces of germs of holomorphic functions. As a byproduct, we get a new 
proof of a celebrated result due to Gabrielov [11]: for an $A:\left(\mathbb{C}^{m}, 0\right) \rightarrow\left(\mathbb{C}^{n}, 0\right)$ which is generically of full rank $n$, if $f \circ A$ is convergent for some formal power series $f$, than $f$ is necessarily convergent, or equivalently, every formal relation between analytic functions $\left(A_{1}, \ldots, A_{n}\right)$ is necessarily analytic (under the assumption that the rank of $A=$ $\left(A_{1}, \ldots, A_{n}\right)$ is generically full). Our Theorem 5 allows us to write an inductive formula for $f$ given $f \circ A$, and we establish the convergence by estimating this induction process directly. We note that this gives an elementary proof of Gabrielov's theorem, which we think is more accessible than the proofs which previously appeared in the literature (see Eakin and Harris [9]).

Let us note that our parametrization works both in the formal and in the analytic setting; and the convergent parametrization can be used to conclude that every formal automorphism between real-analytic CR manifolds which are minimal and holomorphically nondegenerate is convergent. However, these statements are known in greater generality from the work of Baouendi, Mir and Rothschild [5], so we shall refrain from stating them.

\section{Some basics and notation}

This section introduces some basic notation used throughout the paper. Noting that all of the problems considered here are of a local nature, we may (by considering an intrinsic complexification of $M$ ) assume that $M$ is a formal or real analytic generic submanifold of $\mathbb{C}^{N}$, which is defined in normal coordinates $(z, w) \in\left(\mathbb{C}^{n} \times \mathbb{C}^{d}, 0\right)$ by $w=Q(z, \bar{z}, \bar{w})$. Saying that $(z, w)$ are normal coordinates means that $Q$ satisfies

$$
Q(z, 0, \tau)=Q(0, \chi, \tau)=\tau, \quad Q(z, \chi, \bar{Q}(\chi, z, w)) \equiv w .
$$

The first condition makes the coordinates normal, and the second one is referred to as the reality condition.

We study mappings (formal or holomorphic) $H:\left(\mathbb{C}^{n+d}, 0\right) \rightarrow\left(\mathbb{C}^{n+d}, 0\right)$ taking $M$ into $M^{\prime}$, where $M$ and $M^{\prime}$ are generic submanifolds of codimension $d$ in $\mathbb{C}^{n+d}$ defined in normal coordinates as above by $Q$ and $Q^{\prime}$, respectively. We will write $H:(M, 0) \rightarrow$ $\left(M^{\prime}, 0\right)$ if $H$ is such a map taking $M$ into $M^{\prime}$; this is equivalent to the following power series identity:

$$
Q^{\prime}(F(z, Q(z, \chi, \tau)), \bar{H}(\chi, \tau))=G(z, Q(z, \chi, \tau)),
$$

where $H=(F, G)$ is the splitting according to the normal coordinates $\left(z^{\prime}, w^{\prime}\right)$; we often refer to $F$ as the tangential part of $H$ and to $G$ as the transversal part of $H$.

We are now ready to introduce one of the basic tools we will use, the Segre mappings; we have chosen a little variation of the standard notation which is more suitable for our purposes.

\subsection{Iterated Segre mappings}

Assume that we are given a generic manifold $M$ as above defined by $Q$ in normal coordinates $(z, w)$. 
For $j \geq 1$, we define the iterated Segre mappings $S^{j}: \mathbb{C}^{n j} \times \mathbb{C}^{d} \rightarrow \mathbb{C}^{n+d}$ iteratively by the following formulas, where $t \in \mathbb{C}^{d}$ and $x^{k} \in \mathbb{C}^{n}$ for all $k$ :

$$
\begin{aligned}
S^{1}\left(x^{1} ; t\right) & =\left(x^{1}, t\right), \\
S^{j+1}\left(x^{1}, \ldots, x^{j+1} ; t\right) & =\left(x^{1}, Q\left(x^{1}, \bar{S}^{j}\left(x^{2}, \ldots, x^{j+1} ; t\right)\right), \quad j \geq 1 .\right.
\end{aligned}
$$

We also agree to write $S^{0}(t)=(0, t)$, which makes the second line of (5) valid for all $j \geq 0$. We denote by $U^{j}: \mathbb{C}^{n j} \times \mathbb{C}^{d} \rightarrow \mathbb{C}^{d}$ the second component of $S^{j}$, i.e.,

$$
S^{j}\left(x^{1}, \ldots, x^{j} ; t\right)=\left(x^{1}, U^{j}\left(x^{1}, \ldots, x^{j} ; t\right)\right) .
$$

It will be useful to write

$$
x^{[j ; k]}=\left(x^{j}, \ldots, x^{k}\right), \quad j \leq k .
$$

With this notation, the definition of the Segre maps (5) reads

$$
S^{1}\left(x^{1} ; t\right)=\left(x^{1}, t\right), \quad S^{j+1}\left(x^{[1 ; j+1]} ; t\right)=\left(x^{1}, Q\left(x^{1}, \bar{S}^{j}\left(x^{[2 ; j+1]} ; t\right)\right)\right) .
$$

Remark 1. By setting $t=0$ in $S^{j}$, that is, if we consider $S^{j}\left(x^{[1 ; j]} ; 0\right)$, we get the usual iterated Segre maps. However, we need to take derivatives with respect to $t$ before setting $t=0$, which is why we will use a nonstandard notation.

We note the following two useful properties of the Segre maps $S^{j}$ :

$$
\begin{array}{rlrl}
S^{j+1}\left(x^{2}, x^{[1 ; j]} ; t\right) & =S^{j-1}\left(x^{[2 ; j]} ; t\right), & & j \geq 2, \\
S^{2}\left(x^{1}, x^{2} ; \bar{U}^{j}\left(x^{[2 ; j+1]} ; t\right)\right) & =S^{j+1}\left(x^{[1 ; j+1]} ; t\right), & j \geq 2,
\end{array}
$$

where the reality condition (3) has been used to prove (6).

The mapping condition (4) is expressed in terms of the Segre maps as the power series identity

$$
Q^{\prime}\left(F\left(S^{2}(z, \chi ; \tau)\right), \bar{H}(\chi, \tau)\right)=G\left(S^{2}(z, \chi ; \tau)\right) .
$$

By setting $z=x^{1}, \chi=x^{2}$ and $\tau=\bar{U}^{j-1}\left(x^{2}, \ldots, x^{j} ; t\right)$ into (8) and using (7), we get

$$
Q^{\prime}\left(F\left(S^{j}\left(x^{[1 ; j]} ; t\right)\right), \bar{H}\left(\bar{S}^{j-1}\left(x^{[2 ; j]} ; t\right)\right)\right)=G\left(S^{j}\left(x^{[1 ; j]} ; t\right)\right) .
$$

If we instead substitute $z=x^{2}, \chi=x^{1}, \tau=\bar{U}^{j}\left(x^{1}, \ldots, x^{j} ; t\right)$ and use (6) and (7), we get

$$
Q^{\prime}\left(F\left(S^{j-1}\left(x^{[2 ; j]} ; t\right)\right), \bar{H}\left(\bar{S}^{j}\left(x^{[1 ; j]} ; t\right)\right)\right)=G\left(S^{j-1}\left(x^{[2 ; j]} ; t\right)\right) .
$$

Another way of writing the mapping condition (9) is

$$
H\left(S^{j}\left(x^{[1 ; j]} ; t\right)\right) \equiv S^{\prime 2}\left(F\left(S^{j}\left(x^{[1 ; j]} ; t\right)\right), \bar{H}\left(\bar{S}^{j-1}\left(x^{[2 ; j]} ; t\right)\right)\right)
$$

Let $q \geq 2$. By using (10) inductively, together with property (7), we obtain the mapping property of the Segre maps for maps $H:(M, 0) \rightarrow\left(M^{\prime}, 0\right)$, which we refer to as " $H \circ S^{q}=S^{\prime q} \circ H$ ", or more precisely: 
Lemma 3. Let $H:(M, 0) \rightarrow\left(M^{\prime}, 0\right)$ be a mapping. Then

$$
H\left(S^{q}\left(x^{[1 ; q]} ; t\right)\right)=S^{\prime q}\left(y^{[1 ; q]} ; u\right),
$$

where

$$
\begin{gathered}
y^{2 j+1}=F\left(S^{q-2 j}\left(x^{[2 j+1 ; q]} ; t\right)\right), \quad y^{2 j}=\bar{F}\left(S^{q-2 j+1}\left(x^{[2 j ; q]} ; t\right)\right), \\
u= \begin{cases}\bar{G}\left(x^{q}, t\right) & \text { for q even, } \\
G\left(x^{q}, t\right) & \text { for q odd. }\end{cases}
\end{gathered}
$$

\subsection{A lemma on derivatives}

We will need the following lemma, which roughly tells us that if we know the transversal derivatives of a function along a Segre map, then we know all of its derivatives along that map.

Lemma 4. For every $q \in \mathbb{N}, \alpha \in \mathbb{N}^{n}$ and $\beta \in \mathbb{N}^{d}$, there exists a universal power series $p\left(x^{[1 ; q]}, t ; \Lambda\right)$ (depending on $q, \alpha, \beta$, and $M$, convergent if $M$ is), polynomial in its last argument, such that for any power series $h(z, w)$, we have

$$
p\left(x^{[1 ; q]}, t ; \frac{\partial^{|\gamma|}}{\partial\left(x^{1}\right)^{\gamma}} \frac{\partial^{|\delta|}}{\partial t^{\delta}} h\left(S^{q}\left(x^{[1 ; q]} ; t\right)\right)\right),
$$

where $|\gamma|+|\delta| \leq|\alpha|+|\beta|$.

Proof. This is an easy consequence of the chain rule, and the fact that $\frac{\partial U^{j}}{\partial t}$ is invertible near $0 \in \mathbb{C}^{j n+d}$, which follows since $\frac{\partial U^{j}}{\partial t}(0 ; 0)=I_{d \times d}$ by the normality of the coordinates.

\subsection{Jet spaces}

We will need the notion of the jet space along a submanifold. Given the germ of a holomorphic manifold $(X, 0) \subset\left(\mathbb{C}^{r}, 0\right)$, the jet space $J_{X}^{k}\left(\left(\mathbb{C}^{r}, 0\right), \mathbb{C}\right)$ is the quotient of the local ring $\mathcal{O}_{r}$ by the equivalence relation identifying $f \in \mathcal{O}_{r}$ with $g \in \mathcal{O}_{r}$ if

$$
f-g \in I(X)^{k+1},
$$

where

$$
I(X)=\left\{f \in \mathcal{O}_{r}:\left.f\right|_{X}=0\right\}
$$

denotes the ideal associated to $X$. The natural projection $\mathcal{O}_{r} \rightarrow J_{X}^{k}\left(\left(\mathbb{C}^{r}, 0\right), \mathbb{C}\right)$ will be denoted by $j_{X, 0}^{k}$.

In suitable coordinates, we can always assume that $X$ is given by the vanishing of the last, say, $q$ coordinates. In this setting, if $\mathbb{C}^{r}=\mathbb{C}_{x}^{p} \times \mathbb{C}_{t}^{q}$ and we consider jets along $X=\{t=0\}$, we will denote the corresponding jet space by $J_{t}^{k}\left(\mathbb{C}_{x}^{p} \times \mathbb{C}_{t}^{q}, 0\right)$, with the jet map $j_{t, 0}^{k}$. We then have for this choice of coordinates a natural identification

$$
J_{t}^{k}\left(\left(\mathbb{C}_{x}^{p} \times \mathbb{C}_{t}^{q}, 0\right), \mathbb{C}\right)=\left(\mathcal{O}_{p}\right)^{N(k)},
$$


where $N(k)=\sum_{j \leq k}\left(\begin{array}{c}q+j-1 \\ q-1\end{array}\right)$ denotes the number of multiindices in $q$ variables of order at most $k$, given by

$$
j_{t, 0}^{k} h=\left(h(x, 0), \ldots, \frac{\partial^{|\alpha|} h}{\partial t^{\alpha}}(x, 0), \ldots\right),
$$

and we have to include all multiindices $\alpha$ with $|\alpha| \leq k$.

In a similar way we define the jet spaces $J_{X}^{k}\left(\left(\mathbb{C}^{r}, 0\right), \mathbb{C}^{n}\right)$ and $J_{X}^{k}\left(\left(\mathbb{C}^{r}, 0\right),\left(\mathbb{C}^{n}, 0\right)\right)$.

We will use $\varphi(x, t)\left[\left(\alpha_{1}, \alpha_{2}\right)\right]$ to denote the coefficient of $x^{\alpha_{1}} t^{\alpha_{2}}$ in $\varphi(x, t)$, and for any multiindex $\alpha \in \mathbb{N}^{p+q}=\mathbb{N}^{p} \times \mathbb{N}^{q}, \alpha^{1}$ will denote its projection on the first $p$ entries, and $\alpha^{2}$ will denote its projection on the last $q$ entries. For brevity, we sometimes also write $\varphi(x, t)[\alpha]=\varphi_{\alpha}$.

We will be working with the following notion of an "initial term" of a power series $\lambda(x, t)$. We decompose $\lambda$ into homogeneous terms with respect to $x$ and $t$,

$$
\lambda(x, t)=\sum_{j, k} \lambda_{j, k}(x, t), \quad \text { where } \quad \lambda_{j, k}\left(s_{1} x, s_{2} t\right)=s_{1}^{j} s_{2}^{k} \lambda_{j, k}(x, t)
$$

and define the type $\operatorname{tp} \lambda$ of $\lambda$ as the smallest multiindex $\left(n_{1}, n_{2}\right)$ in the lexicographic ordering on $\mathbb{N}^{2}$ which satisfies $\lambda_{n_{1}, n_{2}}(x, t) \not \equiv 0$, i.e.

$$
\lambda_{n_{1}, n_{2}} \not \equiv 0, \quad \text { and } \quad \lambda_{j, k}(x, t)=0 \quad \text { if } k<n_{2} \text { or if } k=n_{2} \text { and } j<n_{1} .
$$

With the notation introduced above, this just means that

$$
\begin{array}{ll}
\lambda\left[\left(\alpha^{1}, \alpha^{2}\right)\right]=0 & \text { if }\left|\alpha^{2}\right|<n_{2}, \\
\lambda\left[\left(\alpha_{0}^{1}, \alpha_{0}^{2}\right)\right] \neq 0 & \text { for some }\left(\alpha_{0}^{1}, \alpha_{0}^{2}\right) \text { with }\left|\alpha_{0}^{1}\right|=n_{1},\left|\alpha_{0}^{2}\right|=n_{2} .
\end{array}
$$

We write

$$
\text { it } \lambda(x, t)=\sum_{\left|\alpha^{1}\right|=n_{1},\left|\alpha^{2}\right|=n_{2}} \lambda_{\left(\alpha^{1}, \alpha^{2}\right)} x^{\alpha^{1}} t^{\alpha^{2}}
$$

and call this the initial term of $\lambda(x, t)$.

\section{Invariants}

Let $(M, 0)$ be a formal generic submanifold, given as usual in normal coordinates by $w=Q(z, \chi, \tau),(z, w) \in \mathbb{C}^{n} \times \mathbb{C}^{d}=\mathbb{C}^{N} ;$ to simplify notation, we write $\zeta=(\chi, \tau)$. In this section, we define an invariant associated to holomorphic nondegeneracy of $(M, 0)$, namely a descending chain of pairs of integers measuring the vanishing of the Segre map (descending in the sense of the lexicographic ordering in $\mathbb{N}^{2}$ ). Recall that holomorphic nondegeneracy of $(M, 0)$ is equivalent to the map

$$
\zeta \mapsto\left(Q_{z^{\alpha}}^{j}(0, \zeta): \alpha \in \mathbb{N}^{n}, 1 \leq j \leq d\right)
$$

being of full rank over the field of meromorphic functions in $\zeta$ (see e.g. [4, Lemma 11.3.5]); again, equivalently, writing $\alpha=\left(\alpha^{1}, \ldots, \alpha^{N}\right)$ and $r=\left(r_{1}, \ldots, r_{N}\right)$, the func- 
tions

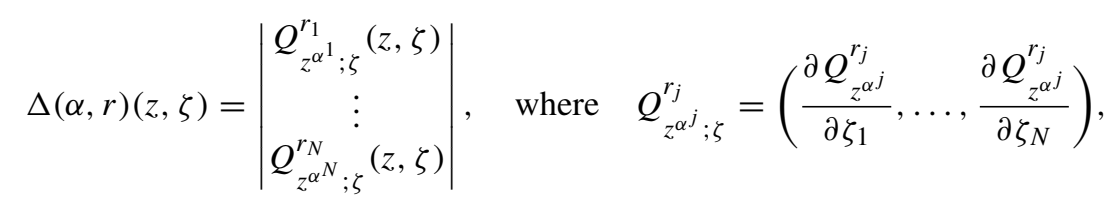

defined for $\alpha \in \mathbb{N}^{n N}, r \in\{1, \ldots, d\}^{N}$, are not all identically zero. To ease the notation, we define the index set $J$ by

$$
J=\mathbb{N}^{(n+d) n} \times\{1, \ldots, d\}^{n+d} .
$$

Now, with the decomposition $\zeta=(\chi, \tau)$ we define the type of a power series $\lambda(\zeta)$, tp $\lambda$, as in (11). Using the lexicographic ordering on $\mathbb{N}^{2}$, we define

$$
\left(n_{1}^{1}, n_{2}^{1}\right)=\min \{\operatorname{tp} \Delta(\alpha, r)(0, \zeta):(\alpha, r) \in J\} .
$$

The higher order version of this invariant is defined for $q \geq 2$ and $(\alpha, r) \in J$ by the functions

$$
\Delta^{q}(\alpha, r)\left(x^{[1 ; q]} ; t\right)=\Delta(\alpha, r)\left(x^{2}, \bar{S}^{q}\left(x^{[1 ; q]} ; t\right)\right)=\left|\begin{array}{c}
Q_{z^{\alpha^{1}} ; \zeta}^{r_{1}}\left(x^{2}, \bar{S}^{q}\left(x^{[1 ; q]} ; t\right)\right) \\
\vdots \\
Q_{z^{\alpha^{N}} ; \zeta}^{r_{N}}\left(x^{2}, \bar{S}^{q}\left(x^{[1 ; q]} ; t\right)\right)
\end{array}\right| ;
$$

again, by holomorphic nondegeneracy, these are not all zero, since

$$
\Delta^{q}(\alpha, r)\left(x_{1}, 0, \ldots, 0 ; t\right)=\Delta(\alpha, r)\left(0, x_{1}, t\right),
$$

and we have already observed that the latter are not all zero.

As above, we define the type of a power series $\lambda(x, t), \operatorname{tp} \lambda$, as in (11). Using the lexicographic ordering on $\mathbb{N}^{2}$, we let

$$
\left(n_{1}^{q}, n_{2}^{q}\right)=\min \left\{\operatorname{tp} \Delta^{q}(\alpha, r)\left(x^{[1 ; q]} ; t\right):(\alpha, r) \in J\right\} .
$$

Let us show that the definition of $\left(n_{1}^{q}, n_{2}^{q}\right)$ for $q \geq 1$ is indeed independent of the choice of normal coordinates. We write $\left(n_{1}^{q}, n_{2}^{q}\right),\left(n_{1}^{\prime q}, n_{2}^{\prime q}\right)$ for the numbers defined by (13) when using $Q$ or $Q^{\prime}$ respectively.

By differentiating the mapping equation $Q^{\prime}(F(z, Q(z, \zeta)), \bar{H}(\zeta))=G(z, Q(z, \zeta))$ with respect to $\zeta$ we obtain

$$
Q_{\zeta^{\prime}}^{\prime}(F(z, Q(z, \zeta)), \bar{H}(\zeta)) \bar{H}_{\zeta}(\zeta)=C_{0}(z, \zeta) Q_{\zeta}(z, \zeta),
$$

where

$$
C_{0}(z, \zeta)=G_{w}(z, Q(z, \zeta))-Q_{z^{\prime}}^{\prime}(F(z, Q(z, \zeta)), \bar{H}(\zeta)) F_{w}(z, Q(z, \zeta)) .
$$

To continue, we first recall 
Lemma 5. Let $(M, 0),\left(M^{\prime}, 0\right)$ be given in normal coordinates by $w=Q(z, \chi, \tau)$ and $w^{\prime}=Q^{\prime}\left(z^{\prime}, \chi^{\prime}, \tau^{\prime}\right)$, respectively, and assume that $H:(M, 0) \rightarrow\left(M^{\prime}, 0\right)$. Then for any $\alpha \in \mathbb{N}^{n}$ with $|\alpha|>0$, there exists a polynomial $P_{\alpha}$ such that

$$
\begin{aligned}
\left(\operatorname{det}\left(\frac{\partial F(z, Q(z, \chi, \tau))}{\partial z}\right)\right)^{2|\alpha|-1} Q_{z^{\prime \alpha}}^{\prime}(F(z, Q(z, \zeta)), \bar{H}(\zeta)) & \\
& =P_{\alpha}\left(\frac{\partial^{|\beta|}}{\partial z^{\beta}} H(z, Q(z, \zeta)):|\beta| \leq|\alpha|\right) .
\end{aligned}
$$

For a multi-index $\alpha$ with $|\alpha|>0$, we deduce from Lemma 5 after differentiation with respect to $\zeta$ that

$$
Q_{z^{\prime \alpha} \zeta}^{\prime}(F(z, Q(z, \zeta)), \bar{H}(\zeta)) \bar{H}_{\zeta}(\zeta)=\sum_{|\beta| \leq|\alpha|} C_{\alpha \beta}(z, \zeta) Q_{z^{\beta} \zeta}(z, \zeta)
$$

where $C_{\alpha \beta}(z, \zeta)$ are some power series. From (14) and (15) we obtain, for any $(\alpha, r) \in J$,

$$
\begin{aligned}
\Delta^{\prime}(\alpha, r)(F(z, Q(z, \zeta)), \bar{H}(\zeta)) \operatorname{det} & \bar{H}_{\zeta}(\zeta) \\
= & \sum_{\substack{(\beta, s) \in J \\
\max \left|\beta^{j}\right| \leq \max \left|\alpha^{j}\right|}} c_{\alpha, r}^{\beta, s}(z, \zeta) \Delta(\beta, s)(z, \zeta),
\end{aligned}
$$

where $c_{\alpha, r}^{\beta, s}(z, \zeta)$ are some power series.

Let us start with the case $q=1$. By substituting $z=0$ into (16), we obtain

$$
\Delta^{\prime}(\alpha, r)(F(0, \tau), \bar{H}(\zeta)) \operatorname{det} \bar{H}_{\zeta}(\zeta)=\sum_{\substack{(\beta, s) \in J \\ \max \left|\beta^{j}\right| \leq \max \left|\alpha^{j}\right|}} c_{\alpha, r}^{\beta, s}(0, \zeta) \Delta^{1}(\beta, s)(\zeta)
$$

By using the identity

$$
\left.\left.\frac{\partial^{|\beta|}}{\partial t^{\beta}}\right|_{t=0} f(x, t, t) \equiv \sum_{\beta^{1}+\beta^{2}=\beta} \frac{\beta !}{\beta^{1} ! \beta^{2} !} \frac{\partial^{|\beta|}}{\partial t^{1^{\beta^{1}}} \partial t^{2^{\beta^{2}}}}\right|_{\substack{t^{1}=0 \\ t^{2}=0}} f\left(x, t^{1}, t^{2}\right),
$$

we get, for any $\gamma$,

$$
\begin{aligned}
\left.\frac{\partial^{|\gamma|}}{\partial \tau^{\gamma}}\right|_{\tau=0} \Delta^{\prime}(\alpha, r)(F(0, \tau), \bar{H}(\chi, \tau))=\left.\frac{\partial^{|\gamma|}}{\partial \tau \gamma}\right|_{\tau=0} \Delta^{\prime}(\alpha, r)(0, \bar{H}(\chi, \tau)) & \\
& +\left.\sum_{\alpha^{\prime}} \sum_{\left|\gamma^{\prime}\right|<|\gamma|} e_{\alpha^{\prime} \gamma^{\prime}} \frac{\partial^{\left|\gamma^{\prime}\right|}}{\partial \tau \gamma^{\prime}}\right|_{\tau=0} \Delta^{\prime}\left(\alpha^{\prime}, r\right)(0, \bar{H}(\chi, \tau)),
\end{aligned}
$$

where we have used that derivatives of $\Delta^{\prime}(\alpha, r)\left(z^{\prime}, \zeta^{\prime}\right)$ with respect to $z^{\prime}$ are linear combinations of $\Delta^{\prime}\left(\alpha^{\prime}, r\right)\left(z^{\prime}, \zeta^{\prime}\right)$ over (finitely) many $\alpha^{\prime}$. Since $\bar{H}(\zeta)$ is invertible and $\bar{G}(\chi, 0)$ $\equiv 0$, we have $\left(n^{\prime}{ }_{1}^{1}, n_{2}^{\prime 1}\right)=\min \left\{\operatorname{tp} \Delta^{\prime}(\alpha, r)(0, \bar{H}(\zeta)):(\alpha, r) \in J\right\}$, so from (18) we obtain $\min \left\{\operatorname{tp} \Delta^{\prime}(\alpha, r)(F(0, \tau), \bar{H}(\chi, \tau)):(\alpha, r) \in J\right\}=\left(n_{1}^{\prime}{ }_{1}, n_{2}^{\prime 1}\right)$. 
Hence, we see from $(17)$, that $\left(n^{\prime}{ }_{1}^{1}, n_{2}^{\prime}{ }_{2}\right) \geq\left(n_{1}^{1}, n_{2}^{1}\right)$ lexicographically; and, by using the inverse mapping to $H$, we obtain the converse inequality, from which we conclude that $\left(n^{\prime \prime}, n^{\prime 1}\right)=\left(n_{1}^{1}, n_{2}^{1}\right)$.

For $q \geq 2$, we substitute $z=x^{2}$ and $\zeta=\bar{S}^{q}\left(x^{[1 ; q]} ; t\right)$ in (16) to obtain

$$
\begin{aligned}
& \Delta^{\prime}(\alpha, r)\left(F\left(S^{q-1}\left(x^{[2 ; q]} ; t\right)\right), \bar{H}\left(\bar{S}^{q}\left(x^{[1 ; q]} ; t\right)\right)\right) \operatorname{det} \bar{H}_{\zeta}\left(\bar{S}^{q}\left(x^{[1 ; q]} ; t\right)\right) \\
& =\sum_{\beta, s} \tilde{c}_{\alpha, r}^{\beta, s}\left(x^{[1 ; q]}, t\right) \Delta^{q}(\beta, s)\left(x^{[1 ; q]} ; t\right)
\end{aligned}
$$

Now Lemma 6 below gives

$$
\min \left\{\operatorname{tp} \Delta^{\prime}(\alpha, r)\left(F\left(S^{q-1}\left(x^{[2 ; q]} ; t\right)\right), \bar{H}\left(\bar{S}^{q}\left(x^{[1 ; q]} ; t\right)\right)\right):(\alpha, r) \in J\right\}=\left(n_{1}^{\prime q}, n_{2}^{\prime q}\right) \text {. }
$$

Hence, we see from (19) that also in this case $\left(n^{\prime}{ }_{1}^{q}, n_{2}^{\prime q}\right) \geq\left(n_{1}^{q}, n_{2}^{q}\right)$.

Again, we can repeat the argument with $H$ replaced with its inverse, so we have $\left(n_{1}^{\prime q}, n_{2}^{\prime q}\right)=\left(n_{1}^{q}, n_{2}^{q}\right)$ for all $q$, and so $\left(n_{1}^{q}, n_{2}^{q}\right)$ are indeed invariants.

Lemma 6. Let $q \geq 2$. With the notation above, we have

$$
\text { it } \Delta^{\prime}(\alpha, r)\left(F\left(S^{q-1}\left(x^{[2 ; q]} ; t\right)\right), \bar{H}\left(\bar{S}^{q}\left(x^{[1 ; q]} ; t\right)\right)\right)=\text { it } \Delta^{\prime q}(\alpha, r)\left(y^{[1 ; q]} ; u\right) \text {, }
$$

where

$$
y^{2 j+1}=\overline{F_{z}(0)} x^{2 j+1}, \quad y^{2 j}=F_{z}(0) x^{2 j}, \quad u= \begin{cases}G_{w}(0) t & \text { for q even }, \\ \overline{G_{w}(0) t} & \text { for } q \text { odd } .\end{cases}
$$

Proof. From Lemma 3, we see that

$$
\Delta^{\prime}(\alpha, r)\left(F\left(S^{q-1}\left(x^{[2 ; q]} ; t\right)\right), \bar{H}\left(\bar{S}^{q}\left(x^{[1 ; q]} ; t\right)\right)\right)=\Delta^{\prime q}(\alpha, r)\left(y^{[1 ; q]} ; u\right),
$$

where

$$
\begin{gathered}
y^{2 j+1}=\bar{F}\left(\bar{S}^{q-2 j}\left(x^{[2 j+1 ; q]} ; t\right)\right), \\
u= \begin{cases}G\left(x^{q}, t\right) & \text { for } q \text { even. } \\
\bar{G}\left(x^{q}, t\right) & \text { for } q \text { odd. }\end{cases}
\end{gathered}
$$

The lemma now follows from the fact that $G(z, 0) \equiv 0$, and that the matrices $F_{z}(0)$ and $G_{w}(0)$ are invertible.

In the course of establishing the invariance, we have actually proved the following stability property of $\left(n_{1}^{q}, n_{2}^{q}\right)$ for $q \geq 1$ for which we will have use later. Here, we use the convention that $S^{q-1}\left(x^{[2 ; q]} ; 0\right)=0$ for $q=1$. 
Lemma 7. Let $(M, 0),\left(M^{\prime}, 0\right)$ be given in normal coordinates by $w=Q(z, \chi, \tau)$ and $w^{\prime}=Q^{\prime}\left(z^{\prime}, \chi^{\prime}, \tau^{\prime}\right)$, respectively, and assume that $H:(M, 0) \rightarrow\left(M^{\prime}, 0\right)$. Then for any choice of $(\alpha, r) \in J$ with tp $\Delta^{\prime q}(\alpha, r)\left(x^{[1 ; q]} ; t\right)=\left(n_{1}^{q}, n_{2}^{q}\right)$ we have tp $\Delta^{\prime}(\alpha, r)\left(F\left(S^{q-1}\left(x^{[2 ; q]} ; t\right)\right), \bar{H}\left(\bar{S}^{q}\left(x^{[1 ; q]} ; t\right)\right)\right)=\left(n_{1}^{q}, n_{2}^{q}\right)$, and

$$
\operatorname{it}\left(\Delta^{\prime}(\alpha, r)\left(F\left(S^{q-1}\left(x^{[2 ; q]} ; t\right)\right), \bar{H}\left(\bar{S}^{q}\left(x^{[1 ; q]} ; t\right)\right)\right)\right)=\left(\text { it } \Delta^{\prime q}(\alpha, r)\right)\left(y^{[1 ; q]}, u\right),
$$

where

$$
y^{2 j+1}=\overline{F_{z}(0)} x^{2 j+1}, \quad y^{2 j}=F_{z}(0) x^{2 j}, \quad u= \begin{cases}G_{w}(0) t & \text { for q even } \\ \overline{G_{w}(0) t} & \text { for } q \text { odd }\end{cases}
$$

\section{A concrete version of Gabrielov's Theorem}

One of the new tools which we use in this paper is the following concrete version and generalization of a theorem of Gabrielov [11]: Given a holomorphic map $A(z):\left(\mathbb{C}^{m}, 0\right) \rightarrow$ $\left(\mathbb{C}^{n}, 0\right)$, where $m \geq n$, we can conclude that $g(w)$ is convergent if $\left(A^{*} g\right)(z)=g(A(z))$ is, provided that the "generic rank" of $A$ is full. This last condition means that the rank of the Jacobian matrix $A^{\prime}(z)$ is full over the field of fractions of $\mathbb{C}\{z\}$. Our Theorem 5 provides a linear inversion of the pullback map $A^{*}: \mathbb{C}[[w]] \rightarrow \mathbb{C}[[z]]$ which restricts to a map $\mathbb{C}\{w\} \rightarrow \mathbb{C}\{z\}$ if $A(z)$ is convergent. Furthermore, our inversion depends holomorphically on $A$ as long as a certain vanishing order associated to $A$ is constant.

For a formal map $A(z):\left(\mathbb{C}^{m}, 0\right) \rightarrow\left(\mathbb{C}^{n}, 0\right)$ we denote by $v(A)$ the minimum order of vanishing of minors of size $n$ of the Jacobian of $A$. To be more precise, we denote $D=\left\{\left(\delta_{1}, \ldots, \delta_{n}\right) \in\{1, \ldots, m\}^{n}: \delta_{1}<\cdots<\delta_{n}\right\}$, and for $\delta \in D$ and a map $A \in \mathcal{H}$, we define

We can thus define

$$
\delta(A)=\left|\begin{array}{ccc}
\frac{\partial A_{1}}{\partial z_{1}} & \cdots & \frac{\partial A_{1}}{\partial z \delta_{n}} \\
\vdots & & \vdots \\
\frac{\partial \dot{A}_{n}}{\partial z_{\delta_{1}}} & \cdots & \frac{\partial A_{n}}{\partial z_{\delta_{n}}}
\end{array}\right| .
$$

$$
v(A)=\min _{\delta \in D} \operatorname{ord} \delta(A)
$$

We write $\mathcal{H}(m, n)$ (resp. $\hat{\mathcal{H}}(m, n))$ for the space of holomorphic (resp. formal holomorphic) maps $\left(\mathbb{C}^{m}, 0\right) \rightarrow\left(\mathbb{C}^{n}, 0\right)$. If the dimensions are clear, we simply write $\mathcal{H}$ or $\hat{\mathcal{H}}$. We can thus consider $v: \hat{\mathcal{H}} \rightarrow \mathbb{N}$; note that the maps of generic full rank are exactly the ones for which $v<\infty$.

Theorem 5. For every $s \geq 0$ there exists a finite family of polynomials $\Psi_{1}, \ldots, \Psi_{\ell(s)}$ on $J_{0}^{s+1}\left(\left(\mathbb{C}^{m}, 0\right),\left(\mathbb{C}^{n}, 0\right)\right)$ and corresponding holomorphic functions

$$
\Phi_{k}(A, f): \hat{U}_{k} \times \mathbb{C}[[z]] \rightarrow \mathbb{C}[[w]], \quad 1 \leq k \leq \ell(s),
$$

where $U_{k}=\left\{A \in \mathcal{H}: \Psi_{k}\left(j_{0}^{s+1} A\right) \neq 0\right\}, \hat{U}_{k}=\left\{A \in \hat{\mathcal{H}}: \Psi_{k}\left(j_{0}^{s+1} A\right) \neq 0\right\}$, and $\Phi_{k}$ is linear in its second variable, such that

$$
\Phi_{k}\left(A, A^{*} g\right)=g \quad \text { for } A \in \hat{U}_{k} \text { with } \nu(A)=s,
$$


and

$$
\bigcup_{k} \hat{U}_{k} \supset\{A \in \hat{\mathcal{H}}: v(A)=s\}, \quad \bigcup_{k} U_{k} \supset\{A \in \mathcal{H}: v(A)=s\}
$$

Furthermore, if $A \in U_{k}$, the operator $\Phi_{k}(A, \cdot)$ restricts to a linear operator $\mathbb{C}\{w\} \rightarrow$ $\mathbb{C}\{z\}$, and the map $\Phi_{k}: U_{k} \times \mathbb{C}\{z\} \rightarrow \mathbb{C}\{w\}$ is holomorphic, where $\mathcal{H}, \mathbb{C}\{w\}$, and $\mathbb{C}\{z\}$ are all equipped with their natural inductive limit topologies.

This theorem is actually a consequence of the following more explicit theorem. We work with the weighted lexicographic ordering on monomials, i.e.

$\alpha \prec \beta$ if $|\alpha|<|\beta|$ or $|\alpha|=|\beta|$ and $\beta-\alpha$ is negative in its first nonvanishing component; correspondingly, for a series $f(z)=\sum_{\alpha} f_{\alpha} z^{\alpha}$ we write

$$
\text { in } f(z)=\min _{\alpha}\left\{f_{\alpha} \neq 0\right\}, \quad \text { it } f(z)=f_{\text {in } f} z^{\text {in } f} .
$$

Theorem 6. For every $\delta \in D$ and every $\delta_{0} \in \mathbb{N}^{m}$, writing $q_{0}=\left|\delta_{0}\right|$ and $\ell(\beta)=$ $|\beta|\left(q_{0}+1\right)$, there exist polynomials $P_{\beta}$ on $J_{0}^{\ell(\beta)}\left(\left(\mathbb{C}^{m}, 0\right),\left(\mathbb{C}^{n}, 0\right)\right) \times J_{0}^{\ell(\beta)}\left(\left(\mathbb{C}^{m}, 0\right), \mathbb{C}\right)$ and a polynomial $c$ on $J_{0}^{q_{0}+1}\left(\left(\mathbb{C}^{m}, 0\right),\left(\mathbb{C}^{n}, 0\right)\right)$ such that if $g(A(z))=f(z)$ and in $\delta(A)=\delta_{0}$, then

$$
g(w)=\sum_{\beta} \frac{P_{\beta}\left(j_{0}^{\ell(\beta)} A, j_{0}^{\ell(\beta)} f\right)}{c\left(j_{0}^{q_{0}+1} A\right)^{2|\beta|-1}} w^{\beta} .
$$

Actually, $c\left(j_{0}^{q_{0}+1} A\right)$ is given by

$$
c\left(j_{0}^{q_{0}+1} A\right)=\frac{\partial^{\left|\delta_{0}\right|} \delta(A)}{\partial z^{\delta_{0}}}(0),
$$

and thus $c\left(j_{0}^{q_{0}+1} A\right) \neq 0$ for all $A$ with in $\delta(A)=\delta_{0}$. Furthermore, for any $M, R>0$, there exist $N, S>0$ such that if $\left|A_{\alpha}\right| \leq M R^{-|\alpha|}$ and $\left|f_{\alpha}\right| \leq M R^{-|\alpha|}$, then

$$
\left|P_{\beta}\left(j_{0}^{\ell(\beta)} A, j_{0}^{\ell(\beta)} f\right)\right| \leq N S^{-|\beta|} .
$$

We split the proof of Theorem 6 in two parts: In 4.1 we discuss how the $P_{\beta}$ are constructed in the formal setting, and we present the estimates in the analytic setting in 4.2. Let us sketch how Theorem 5 follows from Theorem 6: For this, we choose finitely many linear maps $L_{1}, \ldots, L_{\ell^{0}(s)}: \mathbb{C}^{m} \rightarrow \mathbb{C}^{m}$ such that for any homogeneous polynomial $p \in$ $\mathbb{C}\left[z_{1}, \ldots, z_{m}\right]$ of degree $s$ there exists a $k, 1 \leq k \leq \ell^{0}(s)$ with in $\left(p \circ L_{k}\right)=(s, 0, \ldots, 0)$.

We apply Theorem 6 for every $\delta \in D$ and $\delta_{0}=(s, 0, \ldots, 0)$; let us call the corresponding polynomials $P_{\beta}^{\delta}$ and $c_{\delta}$. We define

$$
\Psi_{k, \delta}=\frac{\partial^{s} \delta\left(A \circ L_{k}\right)}{\partial z_{1}^{s}}(0)=c_{\delta}\left(j_{0}^{q_{0}+1}\left(A \circ L_{k}\right)\right) .
$$


Thus, for any $A \in \mathcal{H}$ with $\Psi_{k, \delta}(A) \neq 0$, we have

$$
g(w)=\sum_{\beta} \frac{P_{\beta}^{\delta}\left(j_{0}^{\ell(\beta)}\left(A \circ L_{k}\right), j_{0}^{\ell(\beta)}\left(f \circ L_{k}\right)\right)}{c_{\delta}\left(j_{0}^{q_{0}+1}\left(A \circ L_{k}\right)\right)^{2|\beta|-1}} w^{\beta}=\Phi_{k, \delta}(A, f)(w),
$$

provided that $g(A(z))=f(z)$. The holomorphy of $\Phi_{k, \delta}$ follows from the estimates (21) by the same arguments as presented in $[15, \S 5]$, and Theorem 5 is proved.

\subsection{Formal construction of the $P_{\beta}$}

We assume that $\delta(A)(z) \not \equiv 0$, and write $R^{\delta}(z)$ for the $m \times n$ matrix comprised of the classical adjoint of the columns in $\partial A / \partial z$ corresponding to $\delta$ in the rows corresponding to $\delta$, and 0 in the other rows; thus $A^{\prime}(z) R^{\delta}(z)=\delta(A)(z) I_{n \times n}$. From now on, we will only consider functions $f$ and $g$ such that $f(0)=g(0)=0$, and think about $\delta$ as fixed; we will write $R=R^{\delta}$ and $\lambda(z)=\delta(A)(z)$ for ease of notation.

Given a fixed nonnegative integer $q_{0}$, we define functions $T_{\alpha, \beta}(f, A)$ for $|\alpha| \geq 0$ and $|\beta| \geq 1$, where $f \in \mathbb{C}[[z]], A \in \mathbb{C}[[z]]^{n}$ with $f(0)=0, A(0)=0$ by the following recursion formulas:

$$
\begin{gathered}
T_{\alpha, e_{j}}(f, A)=\sum_{\substack{\alpha^{1}+\alpha^{2}=\alpha \\
1 \leq k \leq m}} \frac{\alpha !}{\alpha^{1} ! \alpha^{2} !} \frac{\partial^{|\alpha|+1} f}{\partial z^{\alpha^{1}+e_{k}}}(0) \frac{\partial^{\left|\alpha^{2}\right|} R_{j}^{k}}{\partial z^{\alpha^{2}}}(0) \\
T_{\alpha, \beta+e_{j}}(f, A)=\sum_{\substack{\left(\alpha^{1}, \alpha^{2}, \alpha^{3}\right) \in I_{\alpha}^{1} \\
1 \leq k \leq m}} \frac{\alpha !}{\alpha^{1} ! \alpha^{2} ! \alpha^{3} !} \frac{\partial^{\left|\alpha^{1}\right|} \frac{\lambda}{\partial z^{\alpha^{1}}}(0) T_{\alpha^{2}+e_{k}, \beta}(f, A) \frac{\partial^{\left|\alpha^{3}\right|} R_{j}^{k}}{\partial z^{\alpha^{3}}}(0)}{-(2|\beta|-1) \sum_{\substack{\left(\alpha^{1}, \alpha^{2}, \alpha^{3}\right) \in I_{\alpha}^{2} \\
1 \leq k \leq m}} \frac{\alpha !}{\alpha^{1} ! \alpha^{2} ! \alpha^{3} !} \frac{\partial^{\left|\alpha^{1}\right|+1} \lambda}{\partial z^{\alpha^{1}+e_{k}}}(0) T_{\alpha^{2}, \beta}(f, A) \frac{\partial^{\left|\alpha^{3}\right|} R_{j}^{k}}{\partial z^{\alpha^{3}}}(0),}
\end{gathered}
$$

where $I_{\alpha}^{1}$ and $I_{\alpha}^{2}$ are index sets defined by

$$
\begin{aligned}
& I_{\alpha}^{1}=\left\{\left(\alpha^{1}, \alpha^{2}, \alpha^{3}\right): \alpha^{1}+\alpha^{2}+\alpha^{3}=\alpha,\left|\alpha^{1}\right| \geq q_{0},\left|\alpha^{2}\right| \geq(2|\beta|-1) q_{0}-1\right\}, \\
& I_{\alpha}^{2}=\left\{\left(\alpha^{1}, \alpha^{2}, \alpha^{3}\right): \alpha^{1}+\alpha^{2}+\alpha^{3}=\alpha,\left|\alpha^{1}\right| \geq q_{0}-1,\left|\alpha^{2}\right| \geq(2|\beta|-1) q_{0}\right\} .
\end{aligned}
$$

We will use the following observation to determine the order $\ell(\beta)$ in Theorem 6 ; the proof is a straightforward induction on $|\beta|$.

Lemma 8. With the notation above, $T_{\alpha, \beta}(f, A)$ is a polynomial in $\frac{\partial^{|\gamma|} f}{\partial z^{\gamma}}(0)$ and $\frac{\partial^{|\gamma|} A}{\partial z^{\gamma}}(0)$ for $|\gamma| \leq|\alpha|-|\beta| q_{0}+|\beta|+q_{0}$, which is linear in $f$. In particular for a $\delta_{0}$ with $\left|\delta_{0}\right|=q_{0}$, $T_{(2|\beta|-1) \delta_{0}, \beta}(f, A)$ is a polynomial in $\frac{\partial^{|\gamma|} f}{\partial z^{\gamma}}(0)$ and $\frac{\partial^{|\gamma|} A}{\partial z^{\gamma}}(0)$ for $|\gamma| \leq|\beta|\left(q_{0}+1\right)$. 
We are now in a position to define the $P_{\beta}$ by

$$
P_{\beta}\left(j_{0}^{\ell(\beta)} A, j_{0}^{\ell(\beta)} f\right)=\frac{T_{(2|\beta|-1) \delta_{0}, \beta}(f, A)}{\beta !\left((2|\beta|-1) \delta_{0}\right) !},
$$

and $c$ by $c\left(j_{0}^{q_{0}+1} A\right)=\frac{\partial^{q_{0}} \lambda(z)}{\partial z_{1}^{q_{0}}}(0)$.

We now need to show that the $P_{\beta}$ defined in that way satisfy (20).

For every multi-index $\beta$ with $|\beta|>0$, we let the function $\psi_{\beta}(z)$ be defined by

$$
\lambda(z)^{2|\beta|-1} g_{w^{\beta}}(A(z))=\psi_{\beta}(z) .
$$

First note that

$$
\psi_{e_{j}}(z)=\sum_{k=1}^{m} f_{z_{k}}(z) R_{j}^{k}(z)
$$

indeed, start by taking the derivative of the equation $g(A(z))=f(z)$ with respect to $z$; this gives $g^{\prime}(A(z)) A^{\prime}(z)=f^{\prime}(z)$, which after application of $R(z)$ reads

$$
\lambda(z) g^{\prime}(A(z))=f^{\prime}(z) R(z) .
$$

If we compare the $j$ th rows in the last equation, we obtain the claimed formula for $\psi_{e_{j}}$. Now we take a derivative of (23), after dividing by $\lambda(z)^{2|\beta|-1}$, and see that

$$
\frac{\partial g_{w^{\beta}}}{\partial w}(A(z)) A^{\prime}(z)=\frac{\lambda(z) \frac{\partial \psi_{\beta}(z)}{\partial z}-(2|\beta|-1) \psi_{\beta}(z) \frac{\partial \lambda(z)}{\partial z}}{\lambda(z)^{2|\beta|}} ;
$$

an application of $R$ leads to

$$
\lambda(z) g_{w^{\beta+e_{j}}}(A(z))=\frac{1}{\lambda(z)^{2|\beta|}} \sum_{k=1}^{m}\left(\lambda(z) \frac{\partial \psi_{\beta}(z)}{\partial z_{k}}-(2|\beta|-1) \psi_{\beta}(z) \frac{\partial \lambda(z)}{\partial z_{k}}\right) R_{j}^{k}(z) .
$$

Thus the $\psi_{\beta}$ satisfy the following recursion formulas:

$$
\psi_{\beta+e_{j}}(z)=\sum_{k=1}^{m}\left(\lambda(z) \frac{\partial \psi_{\beta}(z)}{\partial z_{k}}-(2|\beta|-1) \psi_{\beta}(z) \frac{\partial \lambda(z)}{\partial z_{k}}\right) R_{j}^{k}(z) .
$$

It is now easy to see inductively that $\frac{\partial^{|\alpha|} \psi_{\beta}}{\partial z^{\alpha}}(0)=T_{\alpha, \beta}(f, A)$.

\subsection{Convergence estimates}

To prove the convergence properties, we show that if $A$ is convergent, then the $\psi_{\beta}(z)$ defined in (23) satisfy an estimate of the form $\left|\psi_{\beta}(0)\right| \leq M \beta ! C^{|\beta|}$ for some constants $M$ and $C$, by adapting a method introduced by the first author in his thesis. We start by introducing some notation and stating some lemmata which are needed in the proof of the main estimate in Proposition 11. For a multi-index $\alpha=\left(\alpha_{1}, \ldots, \alpha_{m}\right)$ and a real number $N \geq 0$, we will use $(1+\alpha)^{N}$ to denote $\prod_{j=1}^{m}\left(1+\alpha_{j}\right)^{N}$. 
Lemma 9. Let $N>1$ be a real number. Then there exists a constant $E(N) \leq 2^{N+1} \zeta(N)$ (where $\zeta(N)$ denotes the classical Riemann zeta function) such that for all multi-indices $\alpha=\left(\alpha_{1}, \ldots, \alpha_{m}\right)$,

$$
\sum_{\alpha^{1}+\alpha^{2}=\alpha} \frac{(1+\alpha)^{N}}{\left(1+\alpha^{1}\right)^{N}\left(1+\alpha^{2}\right)^{N}} \leq E(N)^{m} .
$$

Proof. Note that it is enough to treat the case $m=1$; for $m>1$, the estimate follows by rewriting the sum as a product of sums, and applying the estimate for $m=1$ :

$$
\sum_{\alpha^{1}+\alpha^{2}=\alpha} \frac{(1+\alpha)^{N}}{\left(1+\alpha^{1}\right)^{N}\left(1+\alpha^{2}\right)^{N}}=\prod_{j=1}^{n}\left(\sum_{\alpha_{j}^{1}+\alpha_{j}^{2}=\alpha_{j}} \frac{\left(1+\alpha_{j}\right)^{N}}{\left(1+\alpha_{j}^{1}\right)^{N}\left(1+\alpha_{j}^{2}\right)^{N}}\right) \leq E(N)^{m} .
$$

For $m=1$, we estimate as follows:

$$
\begin{aligned}
& \sum_{\alpha^{1}+\alpha^{2}=\alpha} \frac{(1+\alpha)^{N}}{\left(1+\alpha^{1}\right)^{N}\left(1+\alpha^{2}\right)^{N}} \leq 2 \sum_{\ell=0}^{\lfloor\alpha / 2\rfloor} \frac{(1+\alpha)^{N}}{(1+\ell)^{N}(1+\alpha-\ell)^{N}} \\
& \leq 2 \sum_{\ell=0}^{\lfloor\alpha / 2\rfloor} \frac{(1+\alpha)^{N}}{(1+\ell)^{N}(1+\alpha / 2)^{N}} \leq 2^{N+1} \sum_{\ell=0}^{\lfloor\alpha / 2\rfloor} \frac{1}{(1+\ell)^{N}}<2^{N+1} \sum_{\ell=0}^{\infty} \frac{1}{(1+\ell)^{N}}<\infty .
\end{aligned}
$$

We define a useful function $e(\alpha, k)$, where $\alpha=\left(\alpha_{1}, \ldots, \alpha_{m}\right)$ is a multi-index and $k$ is a nonnegative integer in the following way: Choose $p, 1 \leq p \leq m$, such that $\alpha_{p}=\max _{j} \alpha_{j}$ and set

$$
e(\alpha, k)=\left(\alpha_{p}+k\right) ! \prod_{\substack{1 \leq j \leq m \\ j \neq p}} \alpha_{j} !
$$

We will need the following two properties of $e(\alpha, k)$, the proof of which are left to the reader:

Lemma 10. $e(\alpha, k)$, defined in (25), satisfies the following:

$$
\begin{gathered}
e\left(\beta+e_{j}, k\right) \leq e(\beta, k+1), \quad 1 \leq j \leq m, \\
\frac{\alpha ! e\left(\alpha^{1}, k\right)}{\alpha^{1} !} \leq e(\alpha, k), \quad \alpha^{1} \leq \alpha .
\end{gathered}
$$

We can now state the main result of this section.

Proposition 11. Let $N>1$, and assume that

$$
\left|\frac{\partial^{|\alpha|} f}{\partial z^{\alpha}}(0)\right| \leq \alpha ! \frac{M_{1}}{K^{|\alpha|}(1+\alpha)^{N}}, \quad\left|\frac{\partial^{|\alpha|} h}{\partial z^{\alpha}}(0)\right| \leq \alpha ! \frac{M_{2}}{K^{|\alpha|}(1+\alpha)^{N}},
$$

where $h$ denotes any of the functions $\lambda(z), \lambda_{z_{j}}(z)$, or entries of the matrix $R(z)$. There exists a constant $C_{1}=C_{1}\left(K, M_{2}\right)$ such that for all multi-indices $\alpha \in \mathbb{N}^{m}$ and $\beta \in \mathbb{N}^{n}$ with $|\beta|>0$ we have

$$
\left|T_{\alpha, \beta}(f, A)\right| \leq e(\alpha,|\beta|) \frac{M_{1} C_{1}^{|\beta|}}{K^{|\alpha|+|\beta|}(1+\alpha)^{N}} .
$$


Proof. We will prove (27) by induction on $|\beta|$. First, we look at the case $|\beta|=1$, so we estimate, using (26) and Lemma 10:

$$
\begin{aligned}
\left|T_{\alpha, e_{j}}(f, A)\right| & \leq \sum_{k=1}^{m} \sum_{\alpha^{1}+\alpha^{2}=\alpha} \frac{\alpha !}{\alpha^{1} ! \alpha^{2 !}} \frac{\left(\alpha^{1}+e_{k}\right) ! M_{1}}{K^{\left|\alpha^{1}\right|+1}\left(1+\alpha^{1}+e_{k}\right)^{N}} \frac{\left(\alpha^{2}\right) ! M_{2}}{K^{\left|\alpha^{2}\right|}\left(1+\alpha^{2}\right)^{N}} \\
& \leq \frac{M_{1} M_{2}}{K^{|\alpha|+1}} \sum_{k=1}^{m} \sum_{\alpha^{1}+\alpha^{2}=\alpha} \frac{\alpha !}{\alpha^{1} !} e\left(\alpha^{1}, 1\right) \frac{1}{\left(1+\alpha^{1}\right)^{N}} \frac{1}{\left(1+\alpha^{2}\right)^{N}} \\
& \leq \frac{m M_{1} M_{2}}{K^{|\alpha|+1}} e(\alpha, 1) \sum_{\alpha^{1}+\alpha^{2}=\alpha} \frac{1}{\left(1+\alpha^{1}\right)^{N}} \frac{1}{\left(1+\alpha^{2}\right)^{N}} \\
& \leq e(\alpha, 1) \frac{M_{1} C_{1}}{K^{|\alpha|+1}(1+\alpha)^{N}},
\end{aligned}
$$

provided that $C_{1} \geq m M_{2} E(N)^{m}$. We now turn to the induction step, and prove (27) holds with $\beta$ replaced by $\beta+e_{j}$ for some $j$, provided that it holds for all $\beta$ of lesser length. To estimate $T_{\alpha, \beta+e_{j}}$, let us write (22) as

$$
T_{\alpha, \beta+e_{j}}(f, A)=\sum_{\substack{\alpha^{1}+\alpha^{2}=\alpha \\\left|\alpha^{1}\right| \geq 2|\beta| q_{0}-1 \\ 1 \leq k \leq m}} \frac{\alpha !}{\alpha^{1} ! \alpha^{2} !} Q_{\alpha^{1}, \beta, k}(f, A) \frac{\partial^{\left|\alpha^{2}\right|} R_{j}^{k}}{\partial z^{\alpha^{2}}}(0),
$$

where $Q_{\alpha, \beta, k}(f, A)=Q_{\alpha, \beta, k}^{1}(f, A)+Q_{\alpha, \beta, k}^{2}(f, A)$ with

$$
\begin{aligned}
& Q_{\alpha, \beta, k}^{1}(f, A)=\sum_{\substack{\alpha^{1}+\alpha^{2}=\alpha \\
\left|\alpha^{1}\right| \geq q_{0} \\
\left|\alpha^{2}\right| \geq(2|\beta|-1) q_{0}-1}} \frac{\alpha !}{\alpha^{1} ! \alpha^{2} !} \frac{\partial^{\left|\alpha^{1}\right|} \lambda}{\partial z^{\alpha^{1}}}(0) T_{\alpha^{2}+e_{k}, \beta}(f, A), \\
& Q_{\alpha, \beta, k}^{2}(f, A)=-(2|\beta|-1) \sum_{\substack{\alpha^{1}+\alpha^{2}=\alpha \\
\left|\alpha^{1}\right| \geq q^{2}-1 \\
\left|\alpha^{2}\right| \geq(2|\beta|-1) q_{0}}} \frac{\alpha !}{\alpha^{1} ! \alpha^{2} !} \frac{\partial^{\left|\alpha^{1}\right|+1} \lambda}{\partial z^{\alpha^{1}+e_{k}}}(0) T_{\alpha^{2}, \beta}(f, A) .
\end{aligned}
$$

We will first establish estimates for $Q_{\alpha, \beta, k}^{1}$ and $Q_{\alpha, \beta, k}^{2}$. Again using (26), and Lemma 10 as at the induction start, we have

$$
\begin{aligned}
\left|Q_{\alpha, \beta, k}^{1}(f, A)\right| & \leq \sum_{\alpha^{1}+\alpha^{2}=\alpha} \frac{\alpha !}{\alpha^{1} ! \alpha^{2} !} \frac{e\left(\alpha^{1}+e_{k},|\beta|\right) M_{1} C_{1}^{|\beta|}}{K^{\left|\alpha^{1}\right|+|\beta|+1}\left(1+\alpha^{1}+e_{k}\right)^{N}} \frac{\alpha^{2} ! M_{2}}{K^{\left|\alpha^{2}\right|}\left(1+\alpha^{2}\right)^{N}} \\
& \leq e(\alpha,|\beta|+1) \frac{M_{1} M_{2} E(N)^{m} C_{1}^{|\beta|}}{K^{|\alpha|+|\beta|+1}(1+\alpha)^{N}} .
\end{aligned}
$$

Similarly, we see that

$$
\left|Q_{\alpha, \beta, k}^{2}(f, A)\right| \leq e(\alpha,|\beta|+1) \frac{2 M_{1} M_{2} C_{1}^{|\beta|} E(N)^{m}}{K^{|\alpha|+|\beta|}(1+\alpha)^{N}},
$$


and combining this for $Q_{\alpha, \beta, k}=Q_{\alpha, \beta, k}^{1}+Q_{\alpha, \beta, k}^{2}$, we have

$$
\left|Q_{\alpha, \beta, k}(f, A)\right| \leq e(\alpha,|\beta|+1) \frac{M_{1} M_{2} C_{1}^{|\beta|} E(N)^{m}(1+2 K)}{K^{|\alpha|+|\beta|+1}(1+\alpha)^{N}} .
$$

Finally, using (28) we estimate

$$
\begin{aligned}
\left|T_{\alpha, \beta+e_{j}}(f, A)\right| & \leq \sum_{k=1}^{m} \sum_{\alpha^{1}+\alpha^{2}+\alpha} \frac{\alpha !}{\alpha^{1 !}} e\left(\alpha^{1},|\beta|+1\right) \frac{M_{1} M_{2}^{2} C_{1}^{|\beta|} E(N)^{m}(1+2 K)}{K^{|\alpha|+|\beta|+1}\left(1+\alpha^{1}\right)^{N}\left(1+\alpha^{2}\right)^{N}} \\
& \leq e(\alpha,|\beta|+1) \frac{M_{1} M_{2}^{2}(1+2 K) C_{1}^{|\beta|} m E(N)^{2 m}}{K^{|\alpha|+|\beta|+1}(1+\alpha)^{N}} \\
& \leq e(\alpha,|\beta|+1) \frac{M_{1} C_{1}^{|\beta|+1}}{K^{|\alpha|+|\beta|+1}(1+\alpha)^{N}}
\end{aligned}
$$

provided that $C_{1} \geq M_{2}^{2}(1+2 K) m E(N)^{2 m}$. We thus obtain (27) with

$$
C_{1} \geq m M_{2} E(N)^{m} \max \left(M_{2} E(N)^{m}(1+2 K), 1\right) .
$$

The proposition implies that

$$
\frac{1}{\left((2|\beta|-1) \delta_{0}\right) ! \beta !}\left|T_{(2|\beta|-1) \delta_{0}, \beta}(f, A)\right| \leq\left(\frac{(n+1)^{2 \delta_{0 m}+1} C_{1}}{K^{2 q_{0}+1}}\right)^{|\beta|} \cdot \frac{K^{q_{0}}}{(n+1)^{\delta_{0 m}}} \cdot M_{1},
$$

where $\delta_{0 m}=\max _{j} \delta_{0 j}$, which in particular implies the last statement of Theorem 6 , the proof of which is thus finished.

\section{A parametrization theorem}

As noted in the introduction, we need a parametrization theorem for solutions to a certain type of singular analytic equations. While these equations usually do not possess any solutions, if they do, the dependence of the solutions on the right hand side of the equations is analytic as well. More specifically, we have the following theorem. We refer the reader back to $\$ 2.3$ for our notation for the jets appearing in its statement.

Theorem 7. Let $P:\left(\mathbb{C}^{r}, 0\right) \rightarrow\left(\mathbb{C}^{r}, 0\right)$ be a holomorphic map, and assume that $\lambda(y)=$ $\operatorname{det} P_{y}(y) \not \equiv 0$. Let $h^{0}(x, t):\left(\mathbb{C}_{x}^{p} \times \mathbb{C}_{t}^{q}, 0\right) \rightarrow\left(\mathbb{C}^{r}, 0\right)$ be a holomorphic map, and write $\lambda \circ h^{0}=\lambda^{0}, g^{0}=P \circ h^{0}$. Furthermore assume that $\lambda^{0}(x, t) \not \equiv 0$, and write $\left(n_{1}, n_{2}\right)=$ tp $\lambda^{0}$ and $k(\ell)=\max \left(2 n_{2}-1, n_{2}+\ell\right)$. Then there exists an integer $k_{0}$, a neighbourhood $U^{0} \subset J^{k_{0}}\left(\left(\mathbb{C}^{p+q}, 0\right),\left(\mathbb{C}^{r}, 0\right)\right)$ of $j_{0}^{k_{0}} h^{0}$, and for every $\ell \geq 0$ a neighbourhood $V_{\ell}^{0} \subset$ $J_{t}^{k(\ell)}\left(\left(\mathbb{C}_{x}^{p} \times \mathbb{C}_{t}^{q}, 0\right), \mathbb{C}^{r}\right)$ of $j_{t, 0}^{k(\ell)} g^{0}$ and a holomorphic mapping $\Phi_{\ell}: U^{0} \times V_{\ell}^{0} \supset W_{\ell} \rightarrow$ $J_{t}^{\ell}\left(\left(\mathbb{C}^{p} \times \mathbb{C}^{q}, 0\right), \mathbb{C}^{r}\right)$, where $U^{0} \times V_{\ell}^{0} \subset W_{\ell}$, with the property that

$$
j_{t, 0}^{\ell} h=\Phi_{\ell}\left(j_{0}^{k_{0}} h, j_{t, 0}^{k(\ell)}(P \circ h)\right) \quad \text { whenever } \quad \operatorname{tp}(\lambda \circ h)=\left(n_{1}, n_{2}\right) .
$$


Following the arguments given in $[15, \S 5]$, we see that Theorem 7 is an immediate consequence of the following, more explicit theorem, whose proof is the main objective of this section.

Theorem 8. Let $P:\left(\mathbb{C}^{r}, 0\right) \rightarrow\left(\mathbb{C}^{r}, 0\right)$ be a holomorphic map, and assume that $\lambda(y)=$ $\operatorname{det} P_{y}(y) \not \equiv 0$. Let $h^{0}(x, t):\left(\mathbb{C}_{x}^{p} \times \mathbb{C}_{t}^{q}, 0\right) \rightarrow\left(\mathbb{C}^{r}, 0\right)$ be a holomorphic map, and write $\lambda \circ$ $h^{0}=\lambda^{0}$. Furthermore assume that $\lambda^{0}(x, t) \not \equiv 0$, and denote $\left(n_{1}, n_{2}\right)=\operatorname{tp} \lambda^{0}$. Then there exists an integer $k_{0}$, a polynomial $e: J^{k_{0}}\left(\left(\mathbb{C}^{p+q}, 0\right),\left(\mathbb{C}^{r}, 0\right)\right) \rightarrow \mathbb{C}$ with $e\left(j_{0}^{k_{0}} h^{0}\right) \neq 0$, and for every $\alpha \in \mathbb{N}^{p+q}$ a set $N_{\alpha} \subset \mathbb{N}^{p+q}$, a polynomial $p_{\alpha}\left(Y, Z_{\alpha}\right)$, where $Z_{\alpha}$ is a variable in $\mathbb{C}^{r\left|N_{\alpha}\right|}$, and an integer $d_{\alpha}$, with the property that for any formal power series $h(x, t)=\sum_{\beta} h_{\beta} x^{\beta^{1}} t^{\beta^{2}}$ with $h(0,0)=0$ and $g(x, t)=\sum_{\beta} g_{\beta} x^{\beta^{1}} t^{\beta^{2}}$ which satisfy

$$
\left.\frac{\partial^{|\gamma|}}{\partial t^{\gamma}}\right|_{t=0} P(h(x, t))=\left.\frac{\partial^{|\gamma|}}{\partial t^{\gamma}}\right|_{t=0} g(x, t), \quad\left|\gamma^{2}\right| \leq \max \left(2 n_{2}-1, n_{2}+\left|\alpha^{2}\right|\right),
$$

and $\operatorname{tp}(\lambda \circ h)=\left(n_{1}, n_{2}\right)$, we have

$$
h_{\alpha}=\frac{p_{\alpha}\left(j_{0}^{k_{0}} h,\left(g_{\beta}\right)_{\beta \in N_{\alpha}}\right)}{e\left(j_{0}^{k_{0}} h\right)^{d_{\alpha}}} .
$$

The sets $N_{\alpha}$ can be chosen to satisfy $N_{\alpha} \subset\left\{\left(\beta^{1}, \beta^{2}\right):\left|\beta^{2}\right| \leq \max \left(\left|\alpha^{2}\right|+n_{2}, 2 n_{2}-1\right)\right.$, $\left.\left|\beta^{1}\right| \leq a+b\left|\alpha^{1}\right|\right\}$ for some integers $a, b \in \mathbb{N}$.

Furthermore, the $p_{\alpha}$ can be chosen in such a way that for every multiradius $R \in \mathbb{R}_{+}^{p+q}$, $N>0$, and every compact subset $L \subset J^{k_{0}}\left(\left(\mathbb{C}^{p+q}, 0\right),\left(\mathbb{C}^{r}, 0\right)\right) \backslash\{e=0\}$ there exists $a$ multiradius $C \in \mathbb{R}_{+}^{p+q}$ and $M>0$, depending on $R, N, L$ and $P$, with the property that

$$
\text { if }\left|g_{\beta}\right| \leq \frac{N}{R^{\beta}}, \beta \in N_{\alpha}, \quad \text { then } \quad\left|\frac{p_{\alpha}\left(\Lambda,\left(g_{\beta}\right)_{\beta \in N_{\alpha}}\right)}{e(\Lambda)^{d_{\alpha}}}\right| \leq \frac{M}{C^{\alpha}} \quad \text { for all } \Lambda \in L .
$$

We note the following useful corollary, which can be deduced from either Theorem 8 or Theorem 7 by arguments analogous to the proof of Corollary 3.2 in [15]. We note that similar statements hold with $X$ replaced by e.g. a smooth or a topological manifold, with the conclusions valid with the appropriate regularity.

Corollary 12. Let $X$ be a complex manifold, and $g(x, t, \omega)$ be a map defined on a neighbourhood of $\left(0,0, \omega_{0}\right) \in \mathbb{C}^{p} \times \mathbb{C}^{q} \times X$. Let $P:\left(\mathbb{C}^{r}, 0\right) \rightarrow\left(\mathbb{C}^{r}, 0\right)$ be a holomorphic map, and assume that $\lambda(y)=\operatorname{det} P_{y}(y) \not \equiv 0$. Let $h^{0}(x, t):\left(\mathbb{C}_{x}^{p} \times \mathbb{C}_{t}^{q}, 0\right) \rightarrow\left(\mathbb{C}^{r}, 0\right)$, write $\lambda \circ h^{0}=\lambda^{0}$ and $g^{0}=P \circ h^{0}$, and assume that $g^{0}(x, t)=g\left(x, t, \omega_{0}\right)$. Furthermore assume that $\lambda^{0}(x, t) \not \equiv 0$. Then there exists an integer $k_{0}$, a neighbourhood $U^{0} \subset J^{k_{0}}\left(\left(\mathbb{C}^{p+q}, 0\right),\left(\mathbb{C}^{r}, 0\right)\right)$ of $j_{0}^{k_{0}} h^{0}$, and for every $\ell \geq 0$ a neighbourhood $V_{\ell}^{0} \subset X$ of $\omega_{0}$ and a holomorphic mapping $\Phi_{\ell}: U^{0} \times V_{\ell}^{0} \supset W_{\ell} \rightarrow J_{t}^{\ell}\left(\left(\mathbb{C}^{p} \times \mathbb{C}^{q}, 0\right), \mathbb{C}^{r}\right)$, where $\left(n_{1}, n_{2}\right)=\operatorname{tp} \lambda^{0}$, which satisfies

$$
j_{t, 0}^{\ell} h=\Phi_{\ell}\left(j_{0}^{k_{0}} h, \omega\right) \quad \text { if } j_{t, 0}^{k(\ell)}(P \circ h)=j_{t, 0}^{k(\ell)}(g(x, t, \omega)) \text { and } \operatorname{tp}(\lambda \circ h)=\left(n_{1}, n_{2}\right) .
$$

Here we again set $k(\ell)=\max \left(2 n_{2}-1, n_{2}+\ell\right)$. 
Proof of Theorem 8. By assumption, $\lambda^{0}=\operatorname{det} \frac{\partial P}{\partial y}\left(h^{0}(x, t)\right) \not \equiv 0$, and we recall that we write $\left(n_{1}, n_{2}\right)=\operatorname{tp} \lambda^{0}$. We write $A(y)$ for the classical adjoint of $\partial P / \partial y$, that is, $A(y)$ is the $r \times r$-matrix satisfying $\frac{\partial P}{\partial y}(y) A(y)=A(y) \frac{\partial P}{\partial y}(y)=\lambda(y) I_{r \times r}$.

We first make a linear change of coordinates in $x$ and a linear change of coordinates in $t$ to ensure that

$$
e_{0}:=\frac{1}{n_{1} ! n_{2} !} \frac{\partial^{n_{1}+n_{2}} \lambda^{0}}{\partial x_{1}^{n_{1}} \partial t_{1}^{n_{2}}}(0) \neq 0
$$

and write $\delta^{1}=\left(n_{1}, 0, \ldots, 0\right) \in \mathbb{N}^{p}, \delta^{2}=\left(n_{2}, 0, \ldots, 0\right) \in \mathbb{N}^{q}$ and $\delta=\left(\delta^{1}, \delta^{2}\right) \in \mathbb{N}^{p+q}$. Similarly, if $h$ is any map with $\operatorname{tp} \lambda \circ h=\operatorname{tp} \lambda^{0}$, we write

$$
e:=\frac{1}{n_{1} ! n_{2} !} \frac{\partial^{n_{1}+n_{2}}(\lambda \circ h)}{\partial x_{1}^{n_{1}} \partial t_{1}^{n_{2}}}(0)
$$

$e$ is a polynomial in the $n_{1}+n_{2}$-jet of $h$ at 0 .

For the remainder of the proof, we recall that we use the following notation. For any multiindex $\alpha \in \mathbb{N}^{p+q}=\mathbb{N}^{p} \times \mathbb{N}^{q}, \alpha^{1}$ denotes its projection on the first $p$ entries, and $\alpha^{2}$ its projection on the last $q$ entries. We write $\varphi(x, t)[\alpha]$ for the coefficient of $x^{\alpha^{1}} t^{\alpha^{2}}$ in $\varphi(x, t)$. For brevity, we sometimes also write $\varphi(x, t)[\alpha]=\varphi_{\alpha}$.

We first define a weight $\omega_{k}(\alpha)=k_{1}\left|\alpha^{1}\right|+k_{2}\left|\alpha^{2}\right|$ by choosing $k=\left(k_{1}, k_{2}\right)$, where $k_{1}$ and $k_{2}$ are positive integers. Let $p_{0}=\omega_{k}(\delta)$, and assume $k_{2} / k_{1}$ is chosen large enough so that $\left|\alpha^{2}\right|<n_{2}+1$ whenever $\omega_{k}(\alpha) \leq p_{0}$. Note that if $\lambda^{0}[\alpha] \neq 0$ and $\omega_{k}(\alpha) \leq p_{0}$ then $\left(\left|\alpha^{1}\right|,\left|\alpha^{2}\right|\right)=\left(n_{1}, n_{2}\right)$. We also introduce a monomial order $\prec$ by

$$
\alpha \prec \beta: \Leftrightarrow\left\{\begin{array}{l}
\omega_{k}(\alpha)<\omega_{k}(\beta), \text { or } \\
\omega_{k}(\alpha)=\omega_{k}(\beta), \text { and } \alpha^{1}<_{\operatorname{lex}} \beta^{1}, \text { or } \\
\omega_{k}(\alpha)=\omega_{k}(\beta), \text { and } \alpha^{1}=\beta^{1}, \text { and } \alpha^{2}<_{\operatorname{lex}} \beta^{2} .
\end{array}\right.
$$

By Taylor's Theorem, we can rewrite the equation

$$
P(h(x, t))=g(x, t)
$$

for any decomposition of $h(x, t)$ as the sum of two terms, say $h=T_{1}+T_{2}$, as

$$
P\left(T_{1}\right)+P_{y}\left(T_{1}\right) T_{2}+R\left(T_{1}, T_{2}\right)=g(x, t),
$$

where $R\left(T_{1}, T_{2}\right)$ is of order at least 2 in $T_{2}$. After multiplying by $A\left(T_{1}\right)$, we obtain

$$
\lambda\left(T_{1}\right) T_{2}=A\left(T_{1}\right)\left(g(x, t)-P\left(T_{1}\right)-R\left(T_{1}, T_{2}\right)\right) .
$$

We are going to exploit (32) iteratively, and start with the formal statement (i.e. we prove the existence of the polynomials $p_{\alpha}$ ). For any given multiindex $\gamma$ with $\omega_{k}(\gamma)>p_{0}$, we let $\ell=\max \left(\left|\gamma^{2}\right|, n_{2}-1\right)$ and write $h=T_{\gamma} h+S_{\gamma} h$, where $T_{\gamma} h=T^{0} h+T_{\gamma}^{1} h$ and

$$
\left(T^{0} h\right)(x, t)=\sum_{\omega_{k}(\alpha) \leq p_{0}} h_{\alpha} x^{\alpha^{1}} t^{\alpha^{2}}, \quad\left(T_{\gamma}^{1} h\right)(x, t)=\sum_{\substack{\omega_{k}(\alpha)>p_{0}, \alpha<\gamma \\\left|\alpha^{2}\right| \leq \ell}} h_{\alpha} x^{\alpha^{1}} t^{\alpha^{2}} .
$$


If $h$ satisfies the hypotheses of the theorem, we substitute $T_{1}=T_{\gamma} h, T_{2}=S_{\gamma} h$ in (32) and compute the coefficient of $x^{\gamma^{1}+\delta^{1}} t^{\gamma^{2}+\delta^{2}}$. On the left hand side, we obtain

$$
\left(\lambda\left(T_{\gamma} h\right) S_{\gamma} h\right)[\gamma+\delta]=\sum_{\alpha+\beta=\gamma+\delta} \lambda\left(T_{\gamma} h\right)[\alpha] S_{\gamma, \ell} h[\beta]=\lambda\left(T_{\gamma} h\right)[\delta] S_{\gamma} h[\gamma]=e h_{\gamma}
$$

Since $\ell \geq n_{2}-1$ and $\omega_{k}(\gamma)>\omega_{k}(\delta)$, the right hand side simplifies to $A\left(T_{\gamma} h\right)(g(x, t)-$ $\left.P\left(T_{\gamma} h\right)\right)$, which is a polynomial in the coefficients $g_{\alpha}$ of $g(x, t)$ with $\alpha \preceq \gamma+\delta,\left|\alpha^{2}\right| \leq$ $\ell+n_{2}$, as well as in $h_{\alpha}$, where $\alpha \prec \gamma$, and $\alpha^{2} \leq \ell$.

We define $k_{0}=\max \left\{\left|\alpha^{1}\right|+\left|\alpha^{2}\right|: \omega_{k}(\alpha) \leq p_{0}\right\}$. Note that we can regard $e=$ $e\left(\left(h_{\alpha}\right)_{\left.\omega_{k}(\alpha) \leq p_{0}\right)}\right)$ as a polynomial on $J_{0}^{k_{0}}\left(\left(\mathbb{C}_{x}^{p} \times \mathbb{C}_{t}^{q}, 0\right), \mathbb{C}^{r}\right)$. Now a simple induction shows that we can define polynomials $p_{\gamma}(Y, Z)$ in the variables $Y=\left(Y_{\alpha}\right)_{\omega_{k}(\alpha) \leq p_{0}}$ and $Z=$ $\left(Z_{\beta}\right)_{\beta \preceq \gamma+\delta,\left|\beta^{2}\right| \leq \ell+n_{2}}$ such that with some numbers $d_{\gamma}$ for any map $h(x, t)$ satisfying the hypotheses of the theorem we have

$$
h_{\gamma}=\frac{p_{\gamma}\left(\left(h_{\alpha}\right)_{\omega_{k}(\alpha) \leq p_{0}},\left(g_{\beta}\right)_{\beta \leq \gamma+\delta,\left|\beta^{2}\right| \leq \ell+n_{2}}\right)}{e\left(\left(h_{\alpha}\right)_{\omega_{k}(\alpha) \leq p_{0}}\right)^{d_{\gamma}}},
$$

as required.

In order to carry out the estimates, we need to be a bit more precise about how we define the polynomials $p_{\gamma}$. To do that, we will use the splitting $T_{\gamma} h=T^{0} h+T_{\gamma}^{1} h$. We rewrite the right hand side of (32) (with $R$ removed, as it does not contribute any terms) by setting $T_{1}=Y_{1}+Y_{2}$ :

$$
\begin{aligned}
A\left(T_{1}\right)\left(g(x, t)-P\left(T_{1}\right)\right)= & A\left(Y_{1}+Y_{2}\right)\left(g(x, t)-P\left(Y_{1}+Y_{2}\right)\right) \\
=A\left(Y_{1}\right)\left(g(x, t)-P\left(Y_{1}\right)\right) & +\sum_{j, k} \frac{\partial A_{j}}{\partial Y^{k}}\left(Y_{1}\right)\left(g^{j}(x, t)-P^{j}\left(Y_{1}\right)\right) Y_{2}^{k} \\
& -\lambda\left(Y_{1}\right) Y_{2}+R_{2}\left(x, t, Y_{1}, Y_{2}\right),
\end{aligned}
$$

where $R_{2}$ is at least of order 2 in $Y_{2}$; we have written $Y=\left(Y^{1}, \ldots, Y^{r}\right)$, and denoted the $j$ th column of $A$ by $A_{j} ; g^{j}, P^{j}$ are the components of $g$ and $P$, respectively. We will replace $Y_{1}$ and $Y_{2}$ by $\left(T^{0} h\right)(x, t)$ and $\left(T_{\gamma}^{1} h\right)(x, t)$, respectively.

To simplify notation, we write

$$
\begin{aligned}
\tilde{A}_{k}(x, t) & =\sum_{j} \frac{\partial A_{j}}{\partial Y^{k}}\left(\left(T^{0} h\right)(x, t)\right)\left(g^{j}(x, t)-P^{j}\left(\left(T^{0} h\right)(x, t)\right)\right) \\
\tilde{J}(x, t) & =\sum_{j} A_{j}\left(\left(T^{0} h\right)(x, t)\right)\left(g^{j}(x, t)-P^{j}\left(\left(T^{0} h\right)(x, t)\right)\right) \\
R_{2}\left(x, t, Y_{1}, Y_{2}\right) & =\sum_{j, k} R_{2, j, k}\left(x, t, Y_{1}, Y_{2}\right) Y_{2}^{j} Y_{2}^{k} .
\end{aligned}
$$


Using this notation, we define the polynomials $p_{\gamma}$ satisfying (33) by the recursion formula

$$
\begin{aligned}
& e \cdot h_{\gamma}=\tilde{J}(x, t)[\gamma+\delta]+\sum_{k} \sum_{\begin{array}{c}
\alpha+\beta=\gamma+\delta \\
\omega_{k}(\beta) \geq p_{0}+1
\end{array}}\left(T_{\gamma}^{1} h^{k}\right)(x, t)[\alpha] \tilde{A}_{k}(x, t)[\beta] \\
& -\sum_{\substack{\alpha+\beta=\gamma+\delta \\
\omega_{k}(\beta) \geq p_{0}}}\left(T_{\gamma}^{1} h\right)(x, t)[\alpha] \lambda\left(\left(T^{0} h\right)(x, t)\right)[\beta]+R_{2}\left(x, t,\left(T^{0} h\right)(x, t),\left(T_{\gamma}^{1} h\right)(x, t)\right)[\gamma+\delta] .
\end{aligned}
$$

Next we turn to the convergence estimates. For this part we will no longer assume that $h$ satisfies (29) and $\operatorname{tp}(\lambda \circ h)=\left(n_{1}, n_{2}\right)$, but we assume that $h$ satisfies the recursion formula (36). We assume the following convergence estimates for some $R \in \mathbb{R}_{+}^{p+q}, S \in \mathbb{R}_{+}^{r}$, and some $N>1$ :

$$
\begin{array}{rlrl}
\left\|\tilde{A}_{k}(x, t)[\alpha]\right\|_{\infty} & \leq \frac{M_{2}}{R^{\alpha}(1+\alpha)^{N}}, & & \left|\alpha^{2}\right| \leq n_{2}+\ell, \\
\|\tilde{J}(x, t)[\alpha]\|_{\infty} \leq \frac{M_{2}}{R^{\alpha}(1+\alpha)^{N}}, & & \left|\alpha^{2}\right| \leq n_{2}+\ell, \\
\left|\lambda\left(T^{0} h(x, t)\right)[\alpha]\right| \leq \frac{M_{2}}{R^{\alpha}(1+\alpha)^{N}}, & & \left|\alpha^{2}\right| \leq n_{2}+\ell, \\
\left\|R_{2, j, k}\left(x, t, T^{0} h(x, t), Y\right)\left[x^{\alpha^{1}} t^{\alpha^{2}} Y^{\eta}\right]\right\|_{\infty} \leq \frac{M_{2}}{R^{\alpha} S^{\eta}(1+\alpha)^{N}}, & & \left|\alpha^{2}\right| \leq n_{2}+\ell .
\end{array}
$$

We also write

$$
N_{0}=\sum_{\substack{\omega_{k}(\alpha)=p_{0} \\ \alpha>\delta}}\left|\lambda\left(T^{0} h(x, t)\right)[\alpha]\right|
$$

We define $e$ from (31). We will regard $N_{0}$ and $e$ as functions on $J_{0}^{k_{0}}\left(\left(\mathbb{C}_{x}^{p} \times \mathbb{C}_{t}^{q}, 0\right), \mathbb{C}^{r}\right)$. In order to have a common notation, we will assume that we choose the neighbourhood $U^{0}$ such that $e$ and $N_{0}$ remain bounded above, and $e$ remains bounded below on it, and we write

$$
\mathbf{e}=\min _{\Lambda \in U^{0}}|e(\Lambda)|, \quad \mathbf{N}_{0}=\max _{\Lambda \in U^{0}}\left|N_{0}(\Lambda)\right|
$$

We assume that by induction we already have

$$
\left.\| T_{\gamma}^{1} h(x, t)\right)[\alpha] \|_{\infty} \leq \frac{M}{C^{\alpha}(1+\alpha)^{N}} \quad \text { for } \alpha \prec \gamma,\left|\alpha^{2}\right| \leq \ell .
$$

We can assume that the multiradius $R$ is of the form

$$
R=(\underbrace{R_{0}^{k_{1}}, \ldots, R_{0}^{k_{1}}}_{p \text { entries }}, \underbrace{R_{0}^{k_{2}}, \ldots, R_{0}^{k_{2}}}_{q \text { entries }})
$$

for some $R_{0}>0$, and we will choose $C$ of the form

$$
C=(C_{0}^{k_{1}}, \underbrace{C_{0}^{k_{1}} / K, \ldots, C_{0}^{k_{1}} / K}_{p-1 \text { entries }}, C_{0}^{k_{2}}, \underbrace{C_{0}^{k_{2}} / K, \ldots, C_{0}^{k_{2}} / K}_{q-1 \text { entries }}),
$$


where $C_{0}$ and $K$ are real numbers satisfying $0<C_{0}<R_{0}$ and $K \geq 1$. We note that by our choice of ordering it then follows that

$$
\frac{C^{\alpha}}{C^{\delta}} \leq \frac{1}{K} \quad \text { for } \omega_{k}(\alpha)=\omega_{k}(\delta), \delta \prec \alpha .
$$

We also note that

$$
\frac{1}{C^{\delta}} \frac{C^{\beta}}{R^{\beta}}=\frac{1}{C_{0}^{p_{0}}} \frac{C^{\beta}}{R^{\beta}} \leq \frac{C_{0}}{R_{0}^{p_{0}+1}} \quad \text { if } \omega_{k}(\beta)>p_{0} .
$$

We furthermore choose $M$ in (37) to be of the form

$$
M=M_{1} C_{0}^{p_{0}}
$$

Now, we will successively restrict the constants $K, M_{1}$, and $C_{0}$ by requiring that they satisfy the following inequalities ( $E$ denotes the constant given by Lemma 9 for $N$ ):

$$
\begin{aligned}
\frac{(1+|\delta|)^{(p+q) N} \mathbf{N}_{0}}{K} & \leq \frac{\mathbf{e}}{4}, \quad C_{0} \frac{M_{2}}{M_{1}} \frac{1}{R_{0}^{2 p_{0}+1}} \leq \frac{\mathbf{e}}{4}, \\
r^{2} M_{1} M_{2} E^{2(p+q)} & \leq \frac{\mathbf{e}}{4}, \quad \frac{(r+1) C_{0} M_{2} E^{p+q}}{R_{0}^{p_{0}+1}} \leq \frac{\mathbf{e}}{4},
\end{aligned}
$$

and

$$
\max _{j} \frac{C_{j}}{R_{j}}+\sum_{|\eta|>0} \frac{\left(C_{0}^{p_{0}} M_{1} E^{p+q}\right)^{|\eta|}}{S^{\eta}} \leq 1 .
$$

Let us now start estimating terms in (36). We start with

$$
\begin{aligned}
\|\tilde{J}(x, t)[\gamma+\delta]\|_{\infty} & \leq \frac{M_{2}}{R^{\gamma+\delta}(1+\gamma+\delta)^{N}}=\frac{M}{C^{\gamma}(1+\gamma+\delta)^{N}} \frac{M_{2}}{M_{1} R_{0}^{p_{0}}} \frac{C^{\gamma}}{C_{0}^{p_{0}} R^{\gamma}} \\
& \leq \frac{M}{C^{\gamma}(1+\gamma)^{N}} \frac{C_{0} M_{2}}{M_{1} R_{0}^{2 p_{0}+1}} \leq \frac{\mathbf{e}}{4} \frac{M}{C^{\gamma}(1+\gamma)^{N}},
\end{aligned}
$$

where we have used (39) and (38).

We continue with $\sum_{\alpha+\beta=\gamma+\delta, \omega_{k}(\beta) \geq p_{0}}\left(T_{\gamma}^{1} h\right)(x, t)[\alpha] \lambda\left(\left(T^{0} h\right)(x, t)\right)[\beta]$, in which we are going to treat the sums over $\beta$ with $\omega_{k}(\beta)=p_{0}$ and with $\omega_{k}(\beta)>p_{0}$ differently. We have 


$$
\begin{aligned}
\sum_{\substack{\alpha+\beta=\gamma+\delta \\
\omega_{k}(\beta)=p_{0}, \delta<\beta}}\left\|\left(T_{\gamma}^{1} h\right)(x, t)[\alpha]\right\|_{\infty}\left|\lambda\left(\left(T^{0} h\right)(x, t)\right)[\beta]\right| \\
\leq \sum \frac{M}{C^{\alpha}(1+\alpha)^{N}}\left|\lambda\left(\left(T^{0} h\right)(x, t)\right)[\beta]\right| \\
\leq \frac{M}{C^{\gamma}(1+\gamma)^{N}} \sum \frac{C^{\gamma}}{C^{\alpha}} \frac{(1+\gamma)^{N}}{(1+\alpha)^{N}}\left|\lambda\left(\left(T^{0} h\right)(x, t)\right)[\beta]\right| \\
\leq \frac{M(1+|\delta|)^{(p+q) N}}{C^{\gamma}(1+\gamma)^{N}} \sum \frac{C^{\beta}}{C^{\delta}}\left|\lambda\left(\left(T^{0} h\right)(x, t)\right)[\beta]\right| \leq \frac{M(1+|\delta|)^{(p+q) N} \mathbf{N}_{0}}{K C^{\gamma}(1+\gamma)^{N}} \\
\leq \frac{\mathbf{e}}{4} \frac{M}{C^{\gamma}(1+\gamma)^{N}}
\end{aligned}
$$

for terms with $\omega_{k}(\beta)=p_{0}$, where we have used (39) in the last line. For the other terms, we use (38) and estimate

$$
\begin{gathered}
\sum_{\substack{\alpha+\beta=\gamma+\delta \\
\omega_{k}(\beta)>p_{0}}}\left\|\left(T_{\gamma}^{1} h\right)(x, t)[\alpha]\right\|_{\infty}\left|\lambda\left(\left(T^{0} h\right)(x, t)\right)[\beta]\right| \leq \sum \frac{M}{C^{\alpha}(1+\alpha)^{N}} \frac{M_{2}}{R^{\beta}(1+\beta)^{N}} \\
\leq \frac{M M_{2}}{C^{\gamma}} \sum \frac{1}{C^{\delta}} \frac{C^{\beta}}{R^{\beta}} \frac{1}{(1+\alpha)^{N}(1+\beta)^{N}} \leq \frac{M}{C^{\gamma}(1+\gamma)^{N}} \frac{C_{0} M_{2} E^{p+q}}{R_{0}^{p_{0}+1}} .
\end{gathered}
$$

We next turn to terms arising from the second term on the right hand side of (36). We can estimate these terms as in (43) by

$$
\begin{aligned}
& \sum_{k} \sum_{\substack{\alpha+\beta=\gamma+\delta \\
\omega_{k}(\beta) \geq p_{0}+1}}\left|\left(T_{\gamma}^{1} h^{k}\right)(x, t)[\alpha]\right|\left\|\tilde{A}_{k}(x, t)[\beta]\right\|_{\infty} \\
& \quad \leq r \sum \frac{M}{C^{\alpha}(1+\alpha)^{N}} \frac{M_{2}}{R^{\beta}(1+\beta)^{N}} \leq \frac{M}{C^{\gamma}(1+\gamma)^{N}} \frac{r C_{0} M_{2} E^{p+q}}{R_{0}^{p_{0}+1}} .
\end{aligned}
$$

If we combine this with the terms from (43), we see that we can estimate them together using (39) by

$$
\frac{M}{C^{\gamma}(1+\gamma)^{N}} \frac{(r+1) C_{0} M_{2} E^{p+q}}{R_{0}^{p_{0}+1}} \leq \frac{\mathbf{e}}{4} \frac{M}{C^{\gamma}(1+\gamma)^{N}} .
$$

Now let us turn to the remaining term in (36) containing $R_{2}$. We write

$$
\left.R_{2, j, k}\left(x, t, T^{0} h(x, t)\right), Y\right)=\tilde{R}_{2, j, k}(x, t, Y)
$$

and claim that

$$
\left\|\tilde{R}_{2, j, k}\left(x, t, T_{\gamma}^{1} h(x, t)\right)[\alpha]\right\|_{\infty} \leq \frac{M_{2}}{C^{\alpha}(1+\alpha)^{N}} .
$$


To prove the claim, we first note that it holds for $\alpha=0$, so we assume $|\alpha|>0$. We estimate

$$
\begin{aligned}
\left\|\tilde{R}_{2, j, k}\left(x, t, T_{\gamma}^{1} h(x, t)\right)[\alpha]\right\|_{\infty} & =\left\|\sum_{\substack{\nu+\sigma=\alpha \\
\nu+\eta>0}} \tilde{R}_{2, j, k}(x, t, Y)\left[x^{\nu^{1}} t^{\nu^{2}} Y^{\eta}\right]\left(T_{\gamma}^{1} h(x, t)\right)^{\eta}[\sigma]\right\|_{\infty} \\
\leq & \frac{M_{2}}{R^{\alpha}(1+\alpha)^{N}}+\sum_{\substack{\nu+\sigma=\alpha \\
|\eta|>0}} \frac{M_{2}}{R^{v} S^{\eta}(1+v)^{N}} \frac{M^{|\eta|} E^{(p+q)(|\eta|-1)}}{(1+\sigma)^{N} C^{\sigma}} .
\end{aligned}
$$

Now, since $C_{j} \leq R_{j}$ and $|\alpha|>0$, we have

$$
\frac{M_{2}}{R^{\alpha}(1+\alpha)^{N}} \leq \frac{M_{2}}{C^{\alpha}(1+\alpha)^{N}} \max _{j} \frac{C_{j}}{R_{j}} .
$$

Again using that $C_{j} \leq R_{j}$, we get

$$
\sum_{\substack{\nu+\sigma=\alpha \\|\eta|>0}} \frac{M_{2}}{R^{v} S^{\eta}(1+v)^{N}} \frac{M^{|\eta|} E^{(p+q)(|\eta|-1)}}{(1+\sigma)^{N} C^{\sigma}} \leq \frac{M_{2}}{C^{\alpha}(1+\alpha)^{N}} \sum_{|\eta|>0} \frac{\left(C_{0}^{p_{0}} M_{1} E^{p+q}\right)^{|\eta|}}{S^{\eta}} .
$$

Hence the claim follows from the assumption (40). We continue to estimate the remainder by

$$
\begin{aligned}
\left\|\tilde{R}_{2, j, k}\left(x, t, T_{\gamma}^{1} h(x, t)\right)\left(T_{\gamma}^{1} h\right)_{j}\left(T_{\gamma}^{1} h\right)_{k}[\gamma+\delta]\right\|_{\infty} & \leq \frac{M_{2} M^{2} E^{2(p+q)}}{C^{\gamma+\delta}(1+\gamma+\delta)^{N}} \\
& \leq \frac{M}{C^{\gamma}(1+\gamma)^{N}} M_{1} M_{2} E^{2(p+q)} .
\end{aligned}
$$

So from (45) and (39), we have

$$
\begin{aligned}
\left\|\sum_{j, k} \tilde{R}_{2, j, k}\left(T_{\gamma}^{1} h\right)_{j}\left(T_{\gamma}^{1} h\right)_{k}[\gamma+\delta]\right\|_{\infty} & \leq \frac{M}{C^{\gamma}(1+\gamma)^{N}} r^{2} M_{1} M_{2} E^{2(p+q)} \\
& \leq \frac{\mathbf{e}}{4} \frac{M}{C^{\gamma}(1+\gamma)^{N}} .
\end{aligned}
$$

We can now combine (41), (42), (44) and (46); we see that with our choices, also

$$
\left|e h_{\gamma}\right| \leq \mathbf{e} \frac{M}{C^{\gamma}(1+\gamma)^{N}},
$$

which ends the proof of the theorem.

\section{Proof of Theorem 2}

After having discussed all the necessary tools, we can finally turn to the proof of Theorem 2. We start with the following proposition, which acts as the induction step in the construction of the parametrization. 
Proposition 13. Let $(M, 0)$ be a generic real-analytic submanifold of $\mathbb{C}^{N}$ which is holomorphically nondegenerate. Then for any $q \geq 1$, there exists a sequence $\left\{m_{q}(\ell)\right\}_{\ell \geq 0}$ of integers with the following property: If for some $\ell \geq 0$, there exists a mapping $\Phi$ defined on some open neighbourhood $U$ of $\{0\} \times V \subset \overline{\mathbb{C}}^{(q-1) n} \times J_{0}^{k}\left(\left(\mathbb{C}^{N}, 0\right),\left(\mathbb{C}^{N}, 0\right)\right)$, taking values in $J_{t}^{m_{q}(\ell)}\left(\left(\mathbb{C}^{(q-1) n} \times \mathbb{C}^{d}, 0\right),\left(\mathbb{C}^{N}, 0\right)\right)$, holomorphic in the first variable and real-analytic in the second, where $V$ is an open neighbourhood of $j_{0}^{k} \mathrm{id}$, such that

$$
j_{t, 0}^{m_{q}(\ell)} H \circ S^{q-1}\left(x^{[2 ; q]}, t\right)=\Phi\left(x^{[2 ; q]}, j_{0}^{k} H\right)
$$

for all $H \in \operatorname{Aut}(M, 0)$ with $j_{0}^{k} H \in V$, then there exists an integer $\tilde{k}$ and a mapping

$$
\Psi: \mathbb{C}^{N} \times J_{0}^{\tilde{k}}\left(\left(\mathbb{C}^{N}, 0\right),\left(\mathbb{C}^{N}, 0\right)\right) \supset \tilde{U} \rightarrow \mathbb{C}^{N}
$$

defined in an open neighbourhood $\tilde{U}$ of $\{0\} \times \tilde{V}$, where $\tilde{V}$ is an open neighbourhood of $j_{0}^{\tilde{k}} \mathrm{id}$, holomorphic in the first variable and real-analytic in the second, which satisfies

$$
j_{t, 0}^{\ell} H \circ S^{q}\left(x^{[1 ; q]}, t\right)=\Psi\left(x^{[1 ; q]}, j_{0}^{k} H\right) \quad \text { for all } H \in \operatorname{Aut}(M, 0) \text { with } j_{0}^{k} H \in \tilde{V} .
$$

Proof. Let us choose normal coordinates for $(M, 0)$ as usual, and choose $(\alpha, r) \in J$ with tp $\Delta^{q}(\alpha, r)=\left(n_{1}^{q}, n_{2}^{q}\right)$. Let us write

$$
\mathcal{Q}(z, \zeta)=\left(Q_{\alpha^{1}}^{r_{1}}(z, \zeta), \ldots, Q_{\alpha^{N}}^{r_{N}}(z, \zeta)\right)
$$

and recall that we define $|\alpha|=\max \left|\alpha^{j}\right|$. Lemma 5 and an application of the chain rule provide us with a mapping $P_{1}$, polynomial in its last argument, such that we can write

$$
\mathcal{Q}(F(z, Q(z, \zeta)), \bar{H}(\zeta))=\frac{P_{1}\left(z, \zeta ; H_{z^{\gamma} w^{\delta}}(z, Q(z, \zeta)):|\gamma|+|\delta| \leq|\alpha|\right)}{\left(\operatorname{det}\left(\frac{\partial F(z, Q(z, \zeta))}{\partial z}\right)\right)^{2|\alpha|-1}} .
$$

Let us write $R\left(z, \zeta ; F_{z}(z, \zeta), F_{w}(z, \zeta)\right)$ for the denominator in (47). In (47), we replace $\zeta$ by $\bar{S}^{q}\left(x^{[1 ; q]} ; t\right)$ and $z$ by $x^{2}$ to obtain

$$
\begin{aligned}
\mathcal{Q}\left(F\left(S^{q-1}\left(x^{[2 ; q]} ; t\right)\right), \bar{H}\left(\bar{S}^{q}\left(x^{[1 ; q]} ; t\right)\right)\right) \\
=\frac{P_{2}\left(x^{[1 ; q]}, t ; H_{z^{\gamma} w^{\delta}}\left(S^{q-1}\left(x^{[2 ; q]} ; t\right)\right):|\gamma|+|\delta| \leq|\alpha|\right)}{R\left(x^{[1 ; q]}, t ; F_{z}\left(S^{q-1}\left(x^{[2 ; q]} ; t\right)\right), F_{w}\left(S^{q-1}\left(x^{[2 ; q]} ; t\right)\right)\right)} .
\end{aligned}
$$

If we choose $m_{q}(\ell) \geq|\alpha|$, we can use the assumption and Lemma 4 applied to the representation of $H \circ S^{q-1}=\Phi$ in order to replace the right hand side of (48) by a fraction $\mathcal{R}\left(x^{[1 ; q]}, t ; j_{0}^{k} H\right)=P\left(x^{[1 ; q]}, t ; j_{0}^{k} H\right) / R\left(x^{[1 ; q]}, t ; j_{0}^{k} H\right)$ satisfying

$$
j_{t, 0}^{m_{q}(\ell)-|\alpha|} \mathcal{Q}\left(F\left(S^{q-1}\left(x^{[2 ; q]} ; t\right)\right), \bar{H}\left(\bar{S}^{q}\left(x^{[1 ; q]} ; t\right)\right)\right)=j_{t, 0}^{m_{q}(\ell)-|\alpha|} \mathcal{R}\left(x^{[1 ; q]}, t ; j_{0}^{k} H\right),
$$

where both $P(x, t ; \Lambda)$ and $R(x, t ; \Lambda)$ are convergent in a neighbourhood of $\{0\} \times J_{0}^{k}\left(\left(\mathbb{C}^{N}, 0\right),\left(\mathbb{C}^{N}, 0\right)\right)$, and $R(0,0 ; \Lambda) \neq 0$ if $\Lambda$ is invertible. We now consider the system of equations

$$
H \circ S^{q-1}=\Phi, \quad \mathcal{Q}\left(F \circ S^{q-1}, \bar{H} \circ \bar{S}^{q}\right)=\mathcal{R},
$$


and we would like to apply Corollary 12 to it. The system is of the form considered there, with $P\left(y_{1}, y_{2}, y_{3}\right)=\left(y_{1}, y_{2}, \mathcal{Q}\left(y_{1}, y_{3}\right)\right)$, so that $\lambda(y)=\Delta(\alpha, r)\left(y_{1}, y_{3}\right)$, with $\Delta$ defined in (12). Corresponding to $H=$ id, we set $h^{0}(x, t)=\left(S^{q-1}\left(x^{[2 ; q]}, t\right), \bar{S}^{q}\left(x^{[1 ; q]}, t\right)\right)$, and we have $\lambda^{0}(x, t)=\lambda \circ h^{0}(x, t)=\Delta^{q}(\alpha, r)\left(x^{[1 ; q]} ; t\right)$, and tp $\lambda^{0}=\left(n_{1}^{q}, n_{2}^{q}\right)$. We now use Corollary 12 with $X=J_{0}^{k}\left(\left(\mathbb{C}^{N}, 0\right),\left(\mathbb{C}^{N}, 0\right)\right)$, setting $g(x, t, \omega)=$ $(\Phi(x, t, \omega), \mathcal{R}(x, t, \omega)), \omega_{0}=j_{0}^{k}$ id. It supplies us with an integer $k_{0}$, a neighbourhood $V_{\ell}^{0}$ of $j_{0}^{k_{0}}$ id, and a holomorphic function $\Phi_{\ell}$ with $j_{t, 0}^{\ell} h=\Phi_{\ell}\left(j_{0}^{k_{0}} h, \omega\right)$ if $h=\left(h_{1}, h_{2}, h_{3}\right)$ satisfies $j_{t, 0}^{k(\ell)} P\left(h_{1}, h_{2}, h_{3}\right)=j_{t, 0}^{k(\ell)} g(x, t, \omega)$. Hence, if we set $m_{q}(\ell)=|\alpha|+k(\ell)$ and use Lemma 7, we see that $\left(h_{1}, h_{2}, h_{3}\right)=\left(F \circ S^{q-1}, G \circ S^{q-1}, \bar{H} \circ \bar{S}^{q}\right)$ satisfies all the assumptions of Corollary 12, and we have, with $\Phi_{\ell}=\left(\Phi_{\ell}^{1}, \Phi_{\ell}^{2}, \Phi_{\ell}^{3}\right)$,

$$
j_{t, 0}^{\ell} \bar{H} \circ \bar{S}^{q}=\Phi_{\ell}^{3}\left(j_{0}^{k_{0}} \bar{H} \circ \bar{S}^{q}, j_{0}^{k} H\right),
$$

and hence we have the required function $\Psi$ with $\tilde{k}=\max \left(k, k_{0}\right)$ after substituting $j_{0}^{k_{0}} \bar{H} \circ \bar{S}^{q}=\varphi\left(j_{0}^{k_{0}} \bar{H}\right)$ and taking the complex conjugate of the last equation.

Corollary 14. Let $(M, 0)$ be a generic real-analytic submanifold of $\mathbb{C}^{N}$ which is holomorphically nondegenerate. Then for any $q \geq 1$, there exists an integer $k(q)$ and a map $\Psi^{q}$ defined on an open neighbourhood of $\{0\} \times V_{q} \subset \mathbb{C}^{q n} \times J_{0}^{k(q)}\left(\left(\mathbb{C}^{N}, 0\right),\left(\mathbb{C}^{N}, 0\right)\right)$ where $V_{q}$ is an open neighbourhood of $j_{0}^{k(q)} \mathrm{id}$, valued in $\mathbb{C}^{N}$, holomorphic in the first variable and real-analytic in the second, such that

$$
H \circ S^{q}\left(x^{[1 ; q]}, 0\right)=\Psi^{q}\left(x^{[1 ; q]}, j_{0}^{k(q)} H\right) \quad \text { for } H \in \operatorname{Aut}(M, 0) \text { with } j_{0}^{k(q)} H \in V_{q}
$$

Furthermore, there exists a polynomial s defined on $J_{0}^{k(q)}\left(\left(\mathbb{C}^{N}, 0\right),\left(\mathbb{C}^{N}, 0\right)\right)$ with $s\left(j_{0}^{k(q)} \mathrm{id}\right) \neq 0$, integers $r_{\alpha}$ and polynomials $p_{\alpha}\left(\right.$ also defined on $\left.J_{0}^{k(q)}\left(\left(\mathbb{C}^{N}, 0\right),\left(\mathbb{C}^{N}, 0\right)\right)\right)$ defined for $\alpha \in \mathbb{N}^{n q}$ such that

$$
\Psi^{q}(x, \omega)=\sum_{\alpha \in \mathbb{N}^{n q}} \frac{p_{\alpha}(\omega)}{s(\omega)^{r_{\alpha}}} x^{\alpha} .
$$

Proof. The corollary follows in a straightforward manner from Proposition 13 after noting that $H(0, t)$ satisfies the assumptions for $q=1$ trivially for any order; in particular, for the order $m_{1}\left(m_{2}\left(\ldots m_{q}(0) \ldots\right)\right)$. Now we can apply the proposition inductively, ending with an integer $k(q)$ as claimed.

In order to check the second part of the corollary, we refer the reader to the specific form of the solution used in the proposition which is constructed in Theorem 8; that is, we use (30) to obtain this result.

Proof of Theorem 2. If $(M, 0)$ is minimal, the Baouendi-Ebenfelt-Rothschild criterion [1] ensures that for some $q \leq d+1$, the Segre map $S^{q}\left(x^{[1 ; d]} ; 0\right)$ is generically of full rank. We can thus apply Theorem 5 to the parametrization of $H$ along $S^{q}$ constructed in Corollary 14. The result now follows immediately. 
Acknowledgments. The first author was supported by the Austrian Science Fund FWF, Projekt P19667.

The second author was supported by the Austrian Federal Ministry of Science and Research BMWF, START Prize AY377.

\section{References}

[1] Baouendi, M. S., Ebenfelt, P., Rothschild, L. P.: Algebraicity of holomorphic mappings between real algebraic sets in $\mathbf{C}^{n}$. Acta Math. 177, 225-273 (1996) Zbl 0890.32005 MR 1440933

[2] Baouendi, M. S., Ebenfelt, P., Rothschild, L. P.: Parametrization of local biholomorphisms of real analytic hypersurfaces. Asian J. Math. 1, 1-16 (1997) Zbl 0943.32021 MR 1480988

[3] Baouendi, M. S., Ebenfelt, P., Rothschild, L. P.: CR automorphisms of real analytic manifolds in complex space. Comm. Anal. Geom. 6, 291-315 (1998) Zbl 0982.32030 MR 1651418

[4] Baouendi, M. S., Ebenfelt, P., Rothschild, L. P.: Real Submanifolds in Complex Space and Their Mappings. Princeton Math. Ser. 47, Princeton Univ. Press, Princeton, NJ (1999) Zbl 0944.32040 MR 1668103

[5] Baouendi, M. S., Mir, N., Rothschild, L. P.: Reflection ideals and mappings between generic submanifolds in complex space. J. Geom. Anal. 12, 543-580 (2002) Zbl 1039.32021 MR 1916859

[6] Cartan, É.: Sur la géométrie pseudo-conforme des hypersurfaces de l'espace de deux variables complexes II. Ann. Scuola Norm. Sup. Pisa Cl. Sci. (2) 1, 333-354 (1932) JFM 58.1256.03 MR 1556687

[7] Cartan, É.: Sur la géométrie pseudo-conforme des hypersurfaces de l'espace de deux variables complexes. Ann. Mat. Pura Appl. 11, 17-90 (1933) Zbl 0005.37304 MR 1553196

[8] Chern, S. S., Moser, J. K.: Real hypersurfaces in complex manifolds. Acta Math. 133, 219271 (1974) Zbl 0302.32015 MR 0709144

[9] Eakin, P. M., Harris, G. A.: When $F(f)$ convergent implies $f$ is convergent. Math. Ann. 229, 201-210 (1977) Zbl 0355.13010 MR 0444651

[10] Ebenfelt, P., Lamel, B., Zaitsev, D.: Finite jet determination of local analytic CR automorphisms and their parametrization by 2-jets in the finite type case. Geom. Funct. Anal. 13, 546-573 (2003) Zbl 1032.32025 MR 1995799

[11] Gabrielov, A. M.: Formal relations between analytic functions. Izv. Akad. Nauk SSSR Ser. Mat. 37, 1056-1090 (1973) (in Russian) Zbl 0297.32007 MR 0346184

[12] Juhlin, R.: Determination of formal CR mappings by a finite jet. Adv. Math. 222, 1611-1648 (2009) Zbl 1179.32005 MR 2555907

[13] Kolár̆, M.: The Catlin multitype and biholomorphic equivalence of models. Int. Math. Res. Notices 2010, 3530-3548 Zbl 1207.32032 MR 2725504

[14] Kolář, M.: Normal forms for hypersurfaces of finite type in $\mathbb{C}^{2}$. Math. Res. Lett. 12, 897-910 (2005) Zbl 1086.32030 MR 2189248

[15] Lamel, B., Mir, N.: Parametrization of local CR automorphisms by finite jets and applications. J. Amer. Math. Soc. 20, 519-572 (2007) Zbl 1112.32017

[16] Stanton, N. K.: Infinitesimal CR automorphisms of rigid hypersurfaces. Amer. J. Math. 117, 141-167 (1995) Zbl 0826.32013 MR 1314461

[17] Tanaka, N.: On the pseudo-conformal geometry of hypersurfaces of the space of $n$ complex variables. J. Math. Soc. Japan 14, 397-429 (1962) Zbl 0113.06303 MR 0145555

[18] Zaitsev, D.: Germs of local automorphisms of real-analytic CR structures and analytic dependence on $k$-jets. Math. Res. Lett. 4, 823-842 (1997) Zbl 0898.32006 MR 1492123 